\title{
Evaluación de asentamientos en el tiempo de sistemas placa-pilote apoyados sobre depósitos arcillosos de Bogotá D.C. utilizando un modelo 3D de elementos finitos
}

\section{Time settlement evaluation of piled-raft systems supported on clayey soils in Bogotá D.C., using 3D FEM}

\section{Juan Sebastián Monzón y Alejandro Varela}

Fecha de entrega: 20 de junio 2017 Fecha de aceptación: 17 de mayo 2018

Grupo de Investigación en Riesgo en Sistemas Naturales y Antrópicos, Facultad Ingeniería, Pontificia Universidad Javeriana, Carrera 7 No. 40-62 - Edificio José Gabriel Maldonado, Bogotá D.C., Colombia, jmonzon@javeriana.edu.co, a.varela@javeriana.edu.co

Este estudio se relaciona con los análisis de sistemas de cimentación placa-pilote apoyados sobre un perfil estratigráfico típico de arcillas en Bogotá D.C. La estratigrafia fue establecida usando alrededor de 57 registros de exploración y 16 ensayos de piezoconos de estudios geotécnicos para la primera línea del Metro proyectado en esta misma ciudad. Se estableció un modelo de 4 capas de suelo y los parámetros geotécnicos para cada una de estas fueron estimados a partir de ensayos de clasificación USCS, peso unitario, corte directo, consolidación unidimensional, compresión inconfinada y triaxiales CU. Basados en estos parámetros geotécnicos, un análisis de FEM en $3 D$ fue realizado para el estudio de un sistema placa-pilote. 54 combinaciones geométricas entre longitud, diámetro, separación entre centros de elementos verticales y espesor de placa, fueron establecidas para la determinación de asentamientos totales teniendo en cuenta el proceso de construcción (300 días) y posterior hasta 20 años para un edificio sin sótanos de 15 pisos. Adicionalmente se estimaron los asentamientos totales usando las metodologías de Placa equivalente, Pilote equivalente, Poulos y Davis, y Poulos-Davis-Randolph para encontrar el número máximo de pilotes necesarios para el cumplimiento del asentamiento máximo a 20 años propuesto por la NSR-10 (2010) - Título H $(30 \mathrm{~cm})$. Finalmente se realizó el análisis de costos globales de construcción para todas las configuraciones geométricas encontradas.

Palabras clave: arcillas de Bogotá D.C., asentamiento total, elementos finitos $3 D$, placa-pilote, Rocscience RS3
This document presents the analyses of a piled-raft system foundation constructed on a typical stratigraphic profile of clays in Bogotá D.C. The stratigraphy was defined using 57 exploration records and 16 piezocone tests from geotechnical studies for the first line of the Metro projected in this city. A 4-layer average soil model was established and the geotechnical parameters for each layer were defined from USCS classification, unit weight, direct shear, unidimensional consolidation, unconfined compression and triaxial $C U$ tests. Based on this geotechnical information, a $3 D$ FEM analysis was performed for this study. 54 geometric combinations between length, diameter, separation between centres of vertical elements and raft thickness, were established to estimate the total settlements taking into account the construction process (300 days) and later on to 20 years for a 15 floors building without basements. In addition, total settlements were estimated using the methodologies of Equivalent Raft, Equivalent Pile, Poulos y Davis, and Poulos-Davis-Randolph to find the maximum number of piles needed to find the 20-year maximum settlement proposed by NSR-10 (2010) Title H (30 cm). Finally, the global construction cost analysis was performed for all the geometrical configurations and settlement methodologies proposed.

Keywords: clays of Bogotá D.C., total settlement, $3 D$ finite elements, piled-raft, Rocscience RS3

\section{Introducción}

El crecimiento urbanístico en las últimas tres décadas alrededor del mundo ha generado un rápido incremento en el número y altura de los edificios (Patil et al., 2013). Para el caso de Bogotá, la demanda inmobiliaria obliga a construir mayores alturas en las áreas de expansión urbana donde 
predominan los subsuelos blandos. Un número importante de estructuras se construyen sobre suelos blandos, por lo que el uso de losas de cimentación combinadas con pilotes (sistemas placa pilote) se ha convertido en la solución más eficiente desde la década de los años setenta, principalmente por el efecto económico favorable sobre otros tipos de cimentación en temas como: incremento de la capacidad portante, reducción de asentamientos totales y diferenciales en la placa (e.g. Fattah et al., 2013; Ibáñez, 2017). Las experiencias exitosas que se tienen a nivel mundial de cimentaciones de edificios de gran altura en sistemas placa-pilotes, se han desarrollado principalmente en arcillas firmes a muy firmes y sobreconsolidadas, como lo son la arcilla de Frankfurt y la arcilla de Londres, así como en depósitos de arenas y margas en Berlín. Por tal razón, es de interés local, establecer si el sistema placapilotes es eficiente en el caso de arcillas de consistencia blanda a media que se encuentran en la ciudad de Bogotá en función del control de asentamientos por consolidación (Palacio, 2015).

En la actualidad existen los siguientes métodos determinísticos de análisis para el cálculo y estimación preliminar de asentamientos de los sistemas placa-pilote, dentro de los cuales están el propuesto por Poulos y Davis (1980), placa equivalente por Tomlinson y Woodward (2014), pilote equivalente por Poulos y Davis (1980) y Poulos-Davis-Randolph, Randolph (1994). De acuerdo con Poulos (2001), dentro de los métodos simplificados, el de mejor acogida internacional es el método de Randolph (1994). Sin embargo, estas metodologías fueron desarrolladas para depósitos arcillosos sobreconsolidados en donde el interés principal son los asentamientos inmediatos, por lo que implícitamente resulta poco relevante la estimación de los asentamientos no elásticos producto de la consolidación (Palacios, 2015). En general las predicciones de asentamientos se realizan para un determinado tiempo después de terminada la construcción de la obra proyectada, 20 años según la NSR-10 (2010). No se evidencia un profundo estudio en publicaciones donde se haga el intento de predecir el comportamiento del asentamiento en años intermedios (Small y Liu, 2008). Aunque se ha puesto una gran atención al asentamiento de un grupo de pilotes y sistemas placa-pilote, se ha prestado poca atención al comportamiento dependiente del tiempo (Fattah et al., 2013).
En vista del vacío existente en los métodos numéricos usados para el análisis de estos sistemas sobre depósitos muy blandos y compresibles, es necesario mejorar y profundizar el entendimiento que se tiene sobre el comportamiento de los asentamientos en el tiempo para sistemas placa-pilote teniendo en cuenta el proceso constructivo (etapas de carga) mediante la metodología de elementos finitos en tres dimensiones y comparar los resultados obtenidos por dicho método con los calculados mediante las metodologías numéricas. Por lo tanto, en este estudio se usó el software RS3 de elementos finitos 3D (rocscience.com), para modelar numéricamente el comportamiento de varios sistemas placa-pilote apoyados sobre los depósitos arcillosos de Bogotá D.C., fue necesario caracterizar el comportamiento geotécnico del subsuelo de la Sabana de Bogotá con ayuda de los estudios geotécnicos preliminares de la primera línea del metro de Bogotá. Fue posible acceder a información de buena calidad, debido a la implementación de nuevas tecnologías para exploración (sondeos CPT y DMT). Además, se llevaron a cabo ensayos de resistencia al corte triaxial que permitieron actualizar la información de los estudios realizados por Moya y Rodríguez (1987), Orozco (2006) y Montaña (2013).

Las condiciones del perfil estratigráfico promedio definido corresponde a dos tipos de suelos: un espesor de suelos desecados superficialmente del orden de $6 \mathrm{~m}$ con intercalaciones de limos. Seguidos por una secuencia de arcillas blandas e intercalaciones de turbas de origen lacustre de la formación Sabana. Esta formación tiene un espesor variable que aumenta desde los piedemontes de los cerros que bordean la Sabana hacia la zona central. En el sector del norte de Bogotá, entre los cerros de Suba y orientales, el espesor máximo encontrado es del orden de $200 \mathrm{~m}$ (Palacios, 2015).

\section{Metodología}

En esta sección se resume la metodología adoptada y condiciones generales del perfil estratigráfico definido para el desarrollo de este estudio. En la superficie se encuentra una capa sobreconsolidada de arcilla firme CL con trazos cafés de materia orgánica con espesor promedio de $6 \mathrm{~m}$, seguido por una arcilla de alta plasticidad $\mathrm{CH}$ gris clara con materia orgánica con espesor variable entre 7.5 a $9 \mathrm{~m}$. Posteriormente hay una capa de arcilla combinada con limos de alta plasticidad $\mathrm{CH}$ gris oscura con materia 
orgánica de espesor promedio de $8 \mathrm{~m}$ y finalmente un material de arcilla de alta plasticidad $\mathrm{CH}$ color café oscuro. Generalmente, la roca meteorizada se encuentra a una profundidad promedio de $113 \mathrm{~m}$. El nivel freático se encuentra entre 2 y $8 \mathrm{~m}$ debajo de la superficie. Bajo esta circunstancia, habitualmente el suelo en superficie tiene una elevada capacidad portante, lo que hace favorable la implementación de sistemas de solo placa, sin embargo, las capas altamente compresibles pueden causar asentamientos excesivos para los edificios cimentados sobre esta. Así entonces, cimentaciones profundas tales como sistemas placa-pilote se deben usar para estas circunstancias.

Se muestra el perfil simplificado y el resumen de las propiedades geomecánicas usadas en el análisis numérico en la Figura 1. La Tabla 1 presenta la media $\mu$, desviación estándar $\sigma$ y covarianza COV del peso unitario $\gamma$, índice de vacíos inicial $e_{0}$ y coeficiente de permeabilidad $k$. La Tabla 2 presenta la media $\mu$, desviación estándar $\sigma$ y covarianza COV de la razón de sobreconsolidación OCR, coeficiente de compresibilidad $C_{c}$ y coeficiente de hinchamiento $C_{r}$. La Tabla 3 presenta la media $\mu$, desviación estándar $\sigma$ y covarianza COV del módulo de Young $E$, la razón de Poisson $v$, el ángulo de fricción interna $\phi$ y la cohesión $c$.

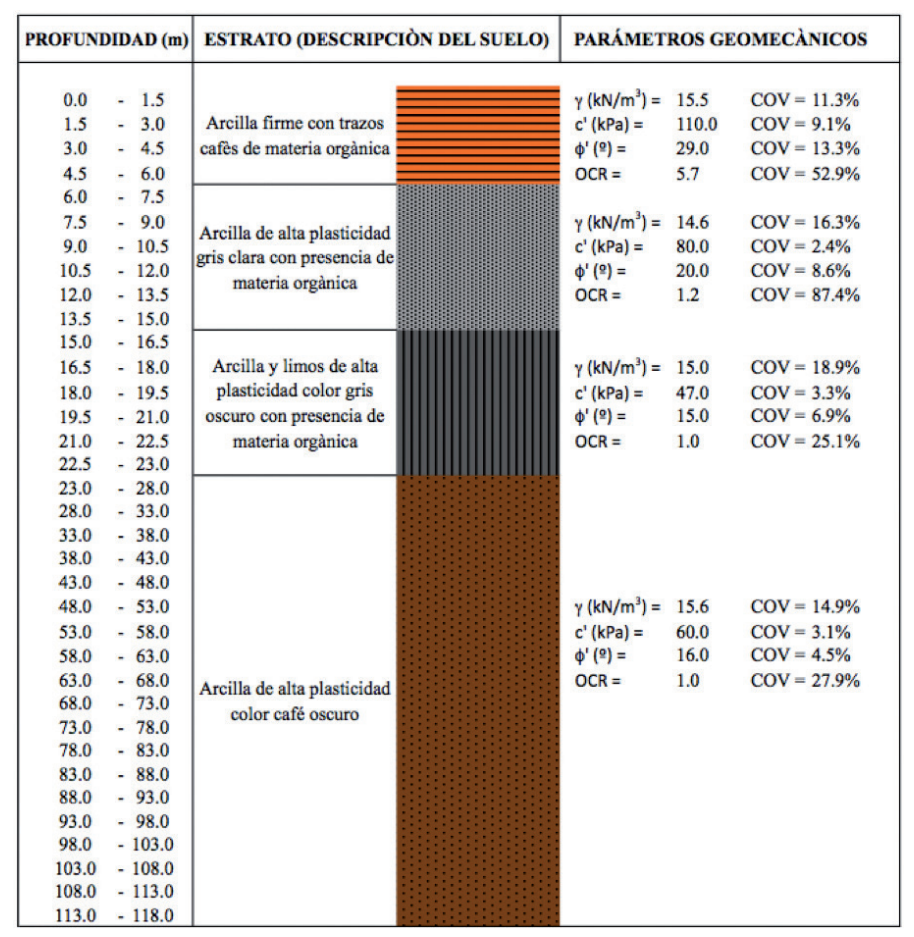

Figura 1: Perfil estratigráfico y parámetros geomecánicos
Tabla 1: Peso unitario $\gamma$, relación de vacíos $e_{0}$ y permeabilidad $k$

\begin{tabular}{|c|c|c|c|c|c|c|c|c|c|}
\hline \multirow{2}{*}{ Capa } & \multicolumn{3}{|c|}{$\gamma, \mathrm{kN} / \mathrm{m}^{3}$} & \multicolumn{4}{c|}{$e_{0}$} & \multicolumn{3}{|c|}{$k, \mathrm{~m} / \mathrm{s} \times 10^{-9}$} \\
\cline { 2 - 10 } & $\mu$ & $\sigma$ & $\begin{array}{c}\mathrm{COV}, \\
\%\end{array}$ & $\mu$ & $\sigma$ & $\begin{array}{c}\mathrm{COV}, \\
\%\end{array}$ & $\mu$ & $\sigma$ & $\begin{array}{c}\mathrm{COV}, \\
\%\end{array}$ \\
\hline 1 & 15.5 & 1.75 & 11.3 & 1.69 & 0.62 & 36.4 & 6.99 & 6.18 & 88.4 \\
\hline 2 & 14.6 & 2.39 & 16.3 & 3.04 & 0.73 & 24.1 & 5.67 & 6.01 & 105.9 \\
\hline 3 & 15.0 & 2.83 & 18.9 & 3.49 & 0.84 & 23.9 & 5.97 & 6.31 & 105.5 \\
\hline 4 & 15.6 & 2.33 & 14.9 & 2.88 & 0.86 & 29.9 & 5.67 & 5.96 & 105.7 \\
\hline
\end{tabular}

Tabla 2: Parámetros de consolidación y compresibilidad

\begin{tabular}{|c|c|c|c|c|c|c|c|c|c|}
\hline \multirow{2}{*}{ Capa } & \multicolumn{3}{|c|}{ OCR } & \multicolumn{4}{c|}{$C_{c}$} & \multicolumn{3}{c|}{$C_{r}$} \\
\cline { 2 - 11 } & $\mu$ & $\sigma$ & $\begin{array}{c}\text { COV, } \\
\%\end{array}$ & $\mu$ & $\sigma$ & $\begin{array}{c}\text { COV, } \\
\%\end{array}$ & $\mu$ & $\sigma$ & $\begin{array}{c}\text { COV, } \\
\%\end{array}$ \\
\hline 1 & 5.69 & 3.02 & 52.9 & 0.71 & 0.33 & 45.8 & 0.134 & 0.10 & 74.9 \\
\hline 2 & 1.16 & 1.43 & 87.4 & 1.46 & 0.69 & 47.1 & 0.267 & 0.31 & 94.5 \\
\hline 3 & 1.01 & 0.26 & 25.1 & 1.67 & 0.80 & 47.9 & 0.268 & 0.09 & 35.5 \\
\hline 4 & 1.01 & 0.28 & 27.9 & 1.97 & 0.39 & 19.6 & 0.189 & 0.19 & 28.7 \\
\hline
\end{tabular}

Tabla 3: Parámetros elásticos y de resistencia

\begin{tabular}{|c|c|c|c|c|c|c|c|c|c|c|c|c|}
\hline \multirow{2}{*}{ Capa } & \multicolumn{3}{|c|}{$E, \mathrm{kPa}$} & \multicolumn{3}{c|}{$v$} & \multicolumn{3}{c|}{$\phi,{ }^{\circ}$} & \multicolumn{3}{c|}{$c, \mathrm{kPa}$} \\
\cline { 2 - 13 } & $\mu$ & $\sigma$ & $\begin{array}{c}\mathrm{COV}, \\
\%\end{array}$ & $\mu$ & $\sigma$ & $\begin{array}{c}\mathrm{COV}, \\
\%\end{array}$ & $\mu$ & $\sigma$ & $\begin{array}{c}\mathrm{COV}, \\
\%\end{array}$ & $\mu$ & $\sigma$ & $\begin{array}{c}\mathrm{COV}, \\
\%\end{array}$ \\
\hline 1 & 7044 & 1786.6 & 25.4 & 0.37 & 0.03 & 7.3 & 29 & 3.8 & 13.3 & 110 & 3.7 & 9.1 \\
\hline 2 & 4367 & 2909.9 & 66.6 & 0.38 & 0.02 & 6.1 & 20 & 1.8 & 8.6 & 80 & 0.7 & 2.4 \\
\hline 3 & 4931 & 3309.3 & 56.1 & 0.38 & 0.03 & 8.8 & 15 & 1.1 & 6.9 & 47 & 1.1 & 3.3 \\
\hline 4 & 5640 & 4103.4 & 51.4 & 0.38 & 0.02 & 4.6 & 16 & 0.7 & 4.5 & 60 & 1.1 & 3.1 \\
\hline
\end{tabular}

Definido el perfil estratigráfico, las propiedades índices y los parámetros geomecánicos, se acoplaron configuraciones geométricas de sistemas de cimentación placa-pilote, definidos en función del equipo constructivo y/o maquinaria comercial disponible en Colombia. La Tabla 4 muestra las configuraciones geométricas dispuesta para los sistemas placa pilote en estudio.

Tabla 4: Configuración geométrica para modelos placa-pilote

\begin{tabular}{|l|c|c|c|}
\hline Geometría de la placa & \multicolumn{3}{|c|}{ Cuadrada de $30 \times 30 \mathrm{~m}$} \\
\hline Diámetro de pilote $D, \mathrm{~m}$ & 0.6 & 0.8 & 1.0 \\
\hline Longitud de pilote $L, \mathrm{~m}$ & 20 & 40 & 60 \\
\hline Separación entre pilotes $s, \mathrm{~m}$ & $2 D$ & $3 D$ & $4 D$ \\
\hline Espesor de placa $h, \mathrm{~m}$ & 0.5 & 1.0 & 1.0 \\
\hline
\end{tabular}

Debido a la simetría del problema, el análisis se limitó a una cuarta parte del sistema placa-pilote como se muestra en la Figura 2. Esto implicó tener en cuenta en el modelo las restricciones y condiciones de frontera suficientes para los bordes de placa y puntos de frontera. 

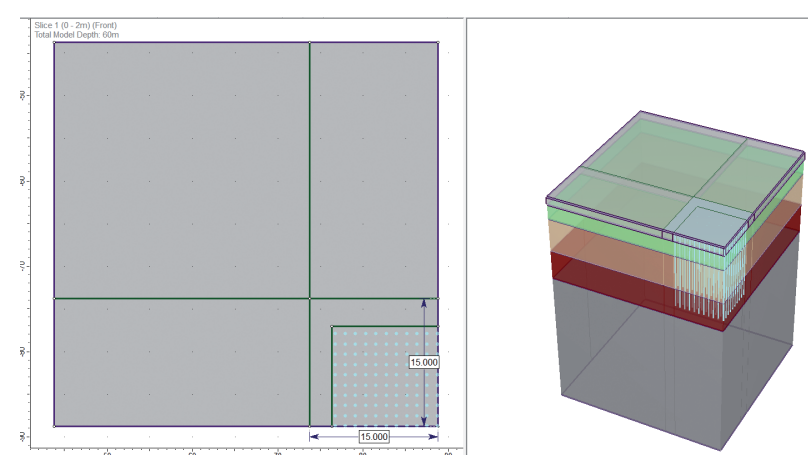

Figura 2: Esquema general de los modelos FEM, cuarta parte del problema

Se estableció que el avance de construcción por piso o planta construida sería en periodos de igual duración. Cada planta se culmina tras un periodo de 20 días por piso, hasta llegar al último nivel de la edificación, lo cual resulta en 15 niveles. La carga muerta de la edificación se calculó suponiendo una carga de $10 \mathrm{kN} / \mathrm{m}^{2}$ por piso. La carga viva fue establecida con base en la información de referencia encontrada en el capítulo de cargas vivas del título B de la NSR-10 (2010), que para uso residencial recomienda usar una carga de $1.8 \mathrm{kN} / \mathrm{m}^{2}$. Por lo tanto, la carga vertical uniformemente distribuida se aplicó en la parte superior de la superficie de la placa después de que se alcanzó el equilibrio inicial. Debido a que la modelación de todo el proceso de instalación de los pilotes es bastante complicada, el pilote se supone que está en un estado de esfuerzo libre al inicio del análisis (Bhowmik y Samanta, 2013; Cho et al., 2012). Por lo tanto, el cambio de esfuerzos en el suelo durante la instalación de los pilotes no fue incluido. Tampoco se tienen en cuenta efectos de excavaciones (alivios de esfuerzos), dado que para el estudio, la placa va estar ubicada en superficie.

Para el cálculo de los asentamientos se definieron dos procesos. El primero de ellos corresponde a la evaluación de los asentamientos durante la construcción de las placas de entrepiso, teniendo en cuenta lo establecido en el párrafo anterior, resultando en un total de 16 etapas constructivas (donde únicamente la carga muerta es aplicada). Finalmente, después de terminada la construcción de cada uno de los pisos, se procede al cálculo de los asentamientos post-construcción (en este cálculo se incluye la carga viva en los análisis), lo cual corresponde al segundo proceso. Dicho proceso consiste de 4 etapas en total, que corresponden al cálculo de los asentamientos para 1, 5, 10 y 20 años después de analizada la construcción. La Tabla 5 muestra el número de etapas simuladas.

Tabla 5: Número de etapas durante y después de finalizada la construcción

\begin{tabular}{|c|c|c|}
\hline Proceso & Etapa & Tiempo, días \\
\hline \multirow[t]{16}{*}{ Durante la construcción } & 1 & 0 \\
\hline & 2 & 20 \\
\hline & 3 & 40 \\
\hline & 4 & 60 \\
\hline & 5 & 80 \\
\hline & 6 & 100 \\
\hline & 7 & 120 \\
\hline & 8 & 140 \\
\hline & 9 & 160 \\
\hline & 10 & 180 \\
\hline & 11 & 200 \\
\hline & 12 & 220 \\
\hline & 13 & 240 \\
\hline & 14 & 260 \\
\hline & 15 & 280 \\
\hline & 16 & 300 \\
\hline \multirow[t]{4}{*}{ Después de la construcción } & 17 & 665 \\
\hline & 18 & 2125 \\
\hline & 19 & 3950 \\
\hline & 20 & 7600 \\
\hline
\end{tabular}

Con el fin de evitar problemas de condiciones de frontera y ahorrar tiempo computacional se usaron las recomendaciones de GGS (2014) sobre las dimensiones mínimas para definir el contorno de los modelos Figura 3.

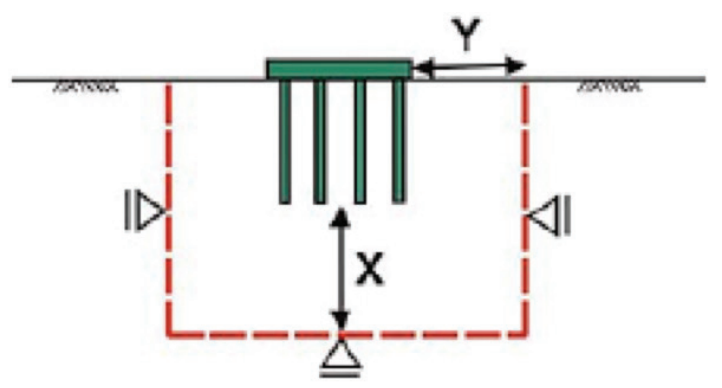

Figura 3: Esquema para el cálculo de dimensiones mínimas para modelos FEM

Donde $\mathrm{X}>1.0$ a 1.5 veces la longitud del pilote e $\mathrm{Y}>1.5 \mathrm{a}$ 2.0 la longitud del pilote. La placa y los pilotes se modelan como un material linealmente elástico. Para el cálculo del módulo de elasticidad $E$ se usó la ecuación presentada en el Título C de la NSR-10 (2010). 


$$
E=3900 \sqrt{f_{c}^{\prime}}
$$

Donde $f_{\mathrm{c}}^{\prime}$ es la resistencia a la compresión a los 28 días con la resistencia característica del concreto $f^{\prime}$ ' para la placa y los pilotes de $28 \mathrm{MPa}$. Las cabezas de los pilotes están conectadas rígidamente a la placa: en la simulación esta conexión se tiene en cuenta por medio de elementos especiales (de un solo nodo) que el programa asigna, lo que asegura que los desplazamientos verticales debajo de la placa sean los mismos.

El programa de elementos finitos $\mathrm{RS}^{3}$ considera dicha interacción mediante la inclusión de una interfaz de un espesor $\mathrm{t}$, determinado entre el pilote y el suelo llamados thin-layer elements y mostrados en la Figura 4. Geométricamente este elemento es similar al elemento continuo (suelo o estructural); sin embargo, sus relaciones constitutivas se definen de manera diferente. El comportamiento normal se define como una función de las propiedades del material y las características de esfuerzodeformación por el elemento continuo vecino. En otras palabras los thin-layer elements son tratados esencialmente como cualquier otro sólido (suelo, roca o estructura) sin embargo su matriz de relación constitutiva (2) es diferente.

$$
\left[C_{i}\right]=\left(\begin{array}{ll}
{\left[C_{n n}\right]_{i}} & {\left[C_{n s}\right]_{i}} \\
{\left[C_{s n}\right]_{i}} & {\left[C_{s s}\right]_{i}}
\end{array}\right)
$$

a)
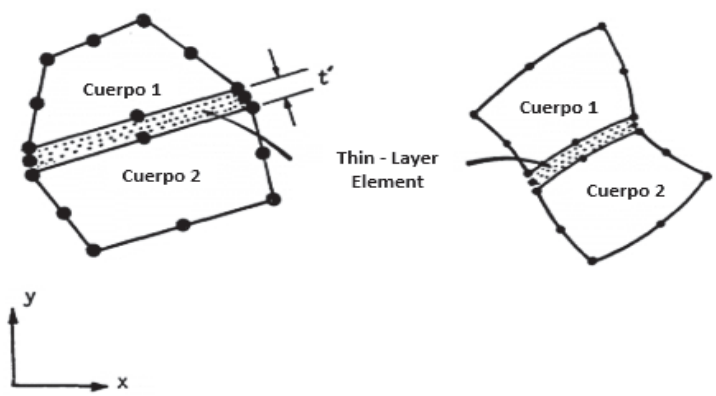

b)

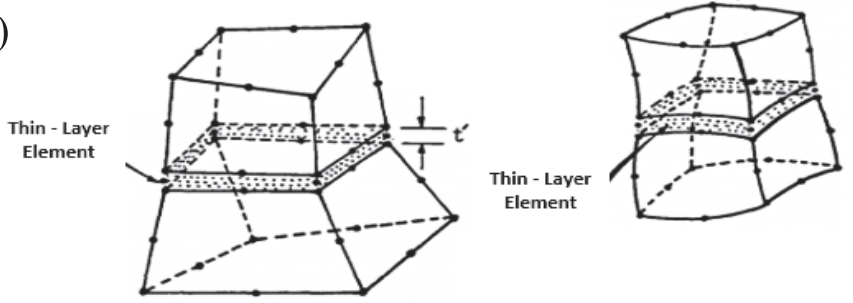

Figura 4: Esquema thin-layer elements, a) en dos dimensión y b) en tres dimensiones
Para definir el comportamiento de esta interfaz es necesario calcular los valores de la rigidez normal y de corte. En la Tabla 6 se muestran los valores de rigidez nomal $K_{n}$ y de corte $K_{s}$ estimados para este estudio. Una guía tentativa propuesta por Desai et al. (1984) para la estimación de la rigidez normal y de corte para los elementos de interfaz thin-layer elements se puede resumir como: a) considerar un espesor promedio del elemento a introducir en la malla y sería denotado como e, b) definir un módulo elástico que se encuentre dentro de los valores de módulo que rodean la interfaz. Si el suelo es estratificado, suponer que es el promedio del módulo de elasticidad de todos los suelos presentes a lo largo del pilote, denotado $E_{q}$, c) calcular la rigidez normal $K_{n}$ dividiendo $E_{q}$ entre el espesor e y d) calcular el valor de la rigidez de corte $K_{s}$ dividiendo $K_{n}$ entre 10 ó 100 .

Para esta investigación el espesor e fue calculado teniendo en cuenta la recomendación propuesta por Desai et al. (1984), en donde la relación entre el espesor e, y la longitud $L$, del elemento debe estar entre 0.01 a 0.1 . Para el estudio la relación fue de 0.01 .

Tabla 6: Valores de rigidez normal $K_{\mathrm{n}} \mathrm{y}$ de corte $K_{\mathrm{s}}$ para cada longitud de pilote

\begin{tabular}{|c|c|c|c|}
\hline$L, \mathrm{~m}$ & $E_{q}, \mathrm{kPa}$ & $K_{n}, \mathrm{kN} / \mathrm{m}$ & $K_{s}, \mathrm{kN} / \mathrm{m}$ \\
\hline 20 & 5312.2 & 26561 & 2656 \\
\hline 40 & 5423.3 & 13558 & 1356 \\
\hline 60 & 5495.6 & 9159 & 916 \\
\hline
\end{tabular}

Los nodos que se encuentran en las esquinas del modelo están restringidos contra desplazamientos en las direcciones $x-y$ para tener el desplazamiento verticalmente. En el plano $x-z$ se restringen los desplazamientos en la dirección $y$ de la misma forma en el plano $y-z$ se restringe el desplazamiento en $x$ y los nodos que constituyen el fondo de la malla son restringidos contra el desplazamiento en las tres direcciones. En la Figura 5 se muestran las restricciones mencionadas.

Los suelos fueron modelados usando el modelo MohrCoulomb. Este modelo fue escogido para representar el comportamiento del suelo, ya que puede predecir el comportamiento de falla del suelo con precisión, aun cuando utiliza parámetros obtenidos de pruebas básicas en laboratorio. El modelo elasto-plástico Mohr- Coulomb 
está basado en una curva de esfuerzo deformación elásticaperfectamente plástica mostrada en la Figura 6.

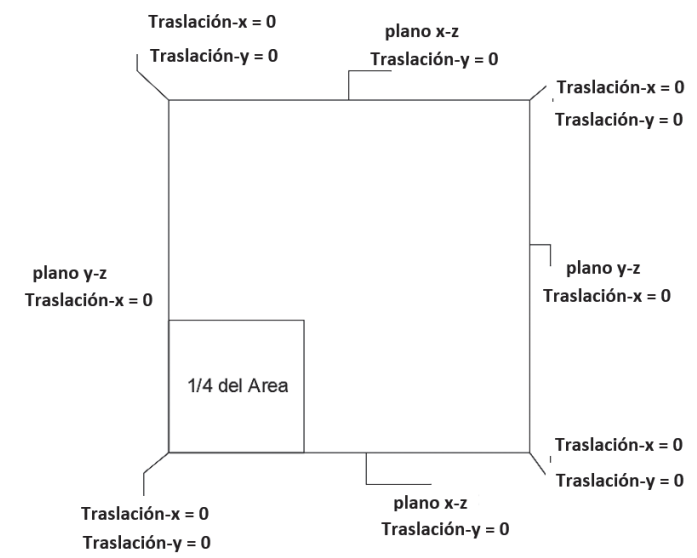

Figura 5: Restricciones de contorno usadas en los modelos FEM

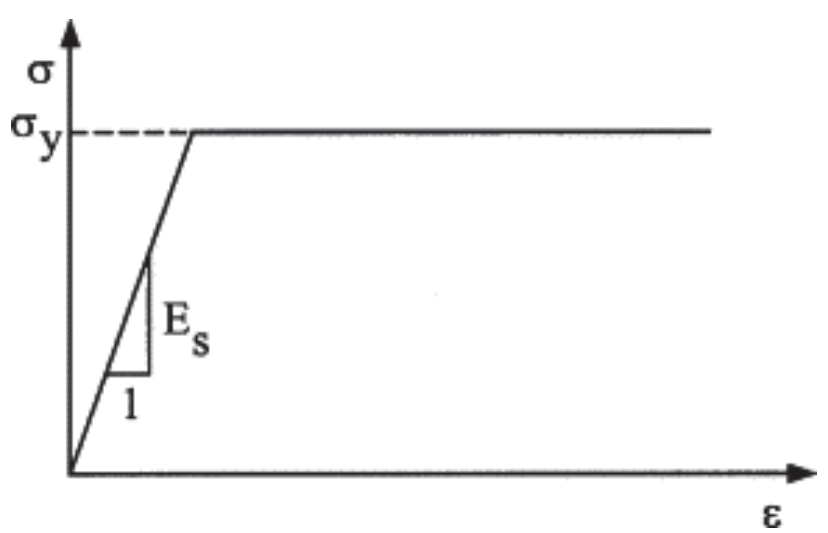

Figura 6: Modelo elástico-perfectamente plástico (Bhowmik y Samanta, 2013)

La característica más atractiva de este modelo es que emplea solo cinco parámetros del suelo, estos son el módulo de Young $E$, la relación de Poisson $v$, la cohesión $c$, el ángulo de fricción $\phi$ y el ángulo de dilatancia $\psi$ para modelar el comportamiento del suelo. Estas propiedades se asumen que permanecen constantes incluso en el momento de ocurrencia del endurecimiento o ablandamiento del material después de la falla. Se usó una malla gruesa, es decir, una malla con elementos muy grandes como se muestra en la Figura 7. Después de garantizar que las cargas y restricciones definidas para el modelo no presentaran ningún tipo de problema, se inició un proceso de refinamiento de la malla, en donde lo que se busca es la resolución del modelo con mallas sucesivamente más finas entre si y comparar los resultados entre estas diferentes mallas. Esta comparación puede hacerse analizando campos en uno o más puntos del modelo, que para el caso de esta investigación es el centro de la placa.

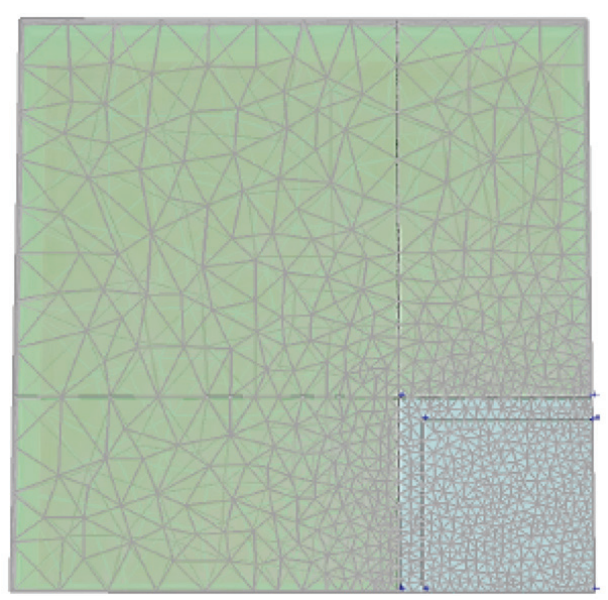

Figura 7: Representación en planta de la malla FEM usada en los modelos

Como criterio de análisis se busca encontrar por medio de las diferentes metodologías analíticas de cálculo y de simulación con elementos finitos, la configuración que cumpla con lo establecido en el numeral H.4.9.2 Límites de asentamientos totales del título H de la NSR10 (2010):"Los asentamientos totales calculados a 20 años se deben limitar al siguiente valor". Para construcciones aisladas $30 \mathrm{~cm}$, siempre y cuando no se afecten la funcionalidad de conducciones y accesos a la construcción. Para el cálculo de los costos y posterior comparación de cada una de las soluciones encontradas, se utilizó un generador de precios de Cype Ingenieros S.A. (Tabla 7).

Tabla 7: Costos globales de construcción para los elementos estructurales en pesos colombianos

\begin{tabular}{|l|c|c|}
\hline Elemento estructural & Unidad & Costo global, $\$$ \\
\hline Pilote $D=0.6 \mathrm{~m}$ & $\mathrm{ml}$ & 309255 \\
\hline Pilote $D=0.8 \mathrm{~m}$ & $\mathrm{ml}$ & 459239 \\
\hline Pilote $D=1.0 \mathrm{~m}$ & $\mathrm{ml}$ & 626018 \\
\hline Placa & $\mathrm{m}^{3}$ & 575661 \\
\hline
\end{tabular}

\section{Resultados}

\section{Método de placa equivalente}

Con el fin de identificar el impacto que tiene la consideración del proceso constructivo (etapas de construcción de cada piso) dentro de la metodología de placa equivalente, se ejecutaron modelos en donde se consideraron ambos escenarios (con proceso y sin proceso). La Figura 8 muestra la evolución de los asentamientos para 1, 5, 10 hasta 20 años. 


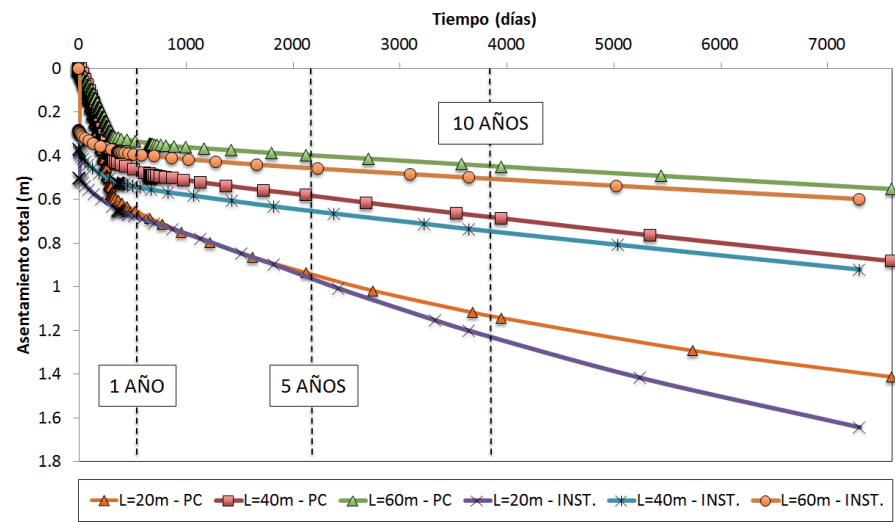

Figura 8: Evolución de asentamientos totales, método Placa equivalente $\sin$ y con proceso constructivo

Se puede observar que los valores de asentamiento obtenidos cuando se consideró el proceso de avance de carga son menores respecto a los del caso sin incremento de las cargas (proceso constructivo). En la Tabla 8 se presentan los valores de asentamientos totales para cada uno de los casos mencionados junto con el porcentaje de variación entre ambas condiciones.

Tabla 8: Variación de asentamientos totales, método Placa equivalente $\sin$ y con proceso constructivo

\begin{tabular}{|c|c|c|c|}
\hline \multirow{2}{*}{$\begin{array}{c}\text { Longitud } \\
\text { de pilotes }\end{array}$ L $\mathrm{m}$} & \multicolumn{2}{|c|}{ Proceso constructivo } & \multirow{2}{*}{ Variación, \% } \\
\cline { 2 - 3 } & $\mathrm{Si}$ & No & \\
\cline { 2 - 3 } & \multicolumn{2}{|c|}{ Asentamiento, $\mathrm{m}$} & \\
\hline 20 & 1.63 & 1.68 & 3 \\
\hline 40 & 0.88 & 0.93 & 4 \\
\hline 60 & 0.55 & 0.60 & 9 \\
\hline
\end{tabular}

La diferencia en los valores obtenidos se debe principalmente al comportamiento en la generación y disipación de excesos de presiones de poros. En la Figura 9 se puede observar que para el caso en donde la carga se aplicó de manera instantánea, el exceso de presión de poros generado fue mayor al que se generó al considerar la aplicación de carga de manera progresiva. A diferencia del comportamiento de rápida disipación de excesos de presiones de poros de la carga instantánea, durante el tiempo que se estableció para la construcción de un piso (20 días x piso), en cada una de las etapas constructivas, se disiparon en promedio $0.9 \mathrm{kPa}$, lo que generó que el valor máximo de exceso de presión de poros sea menor en un rango de $21-34 \%$.
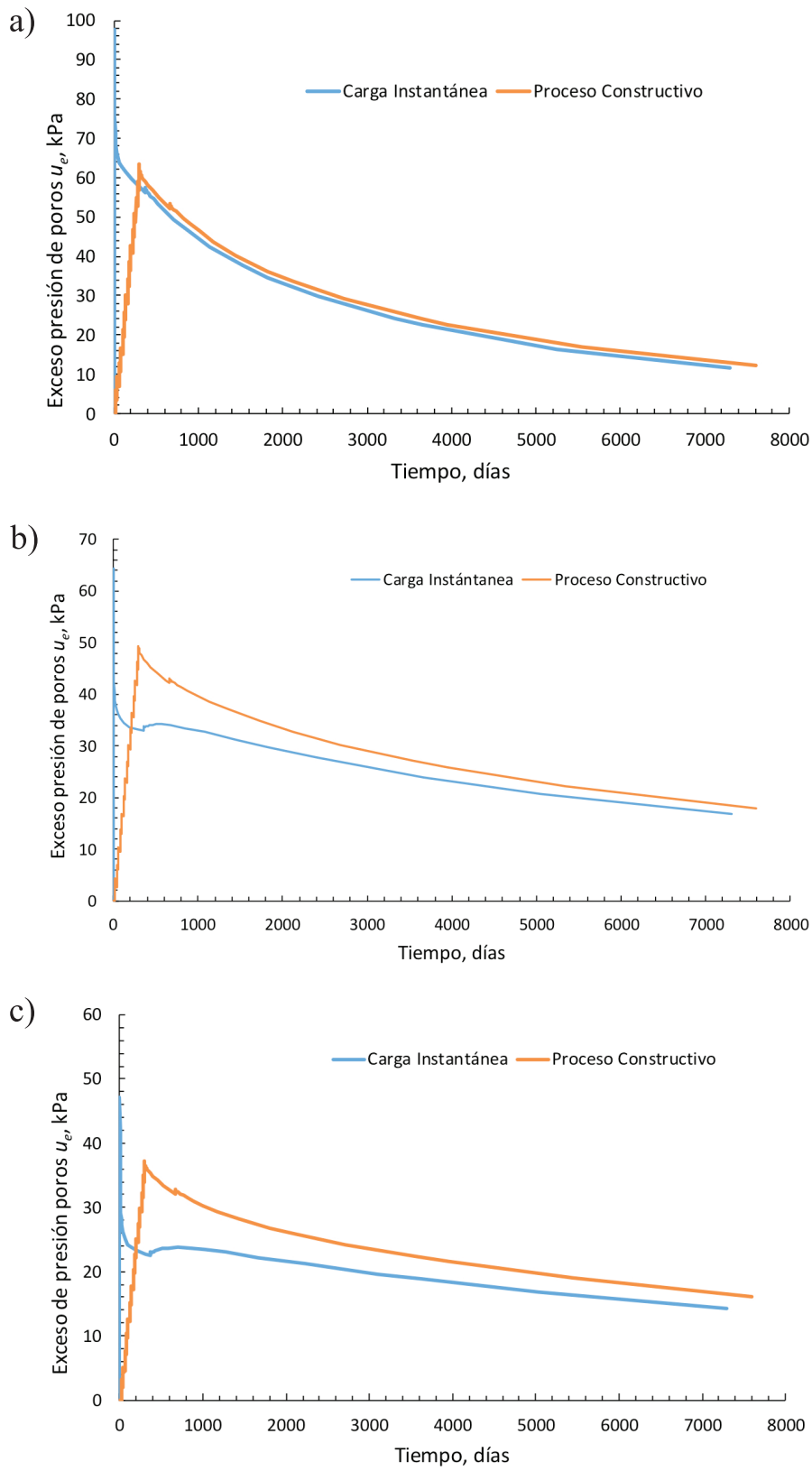

Figura 9: Exceso de presión de poros para una longitud de pilote $L$ de a) $20 \mathrm{~m}$, b) $40 \mathrm{~m}$ y c) $60 \mathrm{~m}$

\section{Método de pilote equivalente}

En las Tablas 9, 10 y 11 se resumen los resultados arrojados por la metodología. Teniendo en cuenta los $30 \mathrm{~cm}$ de asentamiento máximo propuesto en la norma, el $70.4 \%$ de soluciones encontradas cumplen con dicho criterio.

Para el problema propuesto en esta investigación y según los resultados observados anteriormente, los pilotes de longitud de $20 \mathrm{~m}$ sin importar el diámetro y la separación ofrecen un deficiente control de los asentamientos. 
Tabla 9: Número de pilotes y asentamiento total $\delta$ para $D=0.6$ $\mathrm{m}$ (pilote equivalente)

\begin{tabular}{|c|c|c|c|c|}
\hline$L, \mathrm{~m}$ & $S$ & pilotes & $\delta, \mathrm{m}$ & $L / D$ \\
\hline 20 & $2 D$ & 625 & 0.357 & 33.3 \\
\hline 40 & $2 D$ & 300 & 0.300 & 66.7 \\
\hline 60 & $2 D$ & 55 & 0.299 & 100 \\
\hline 20 & $3 D$ & 256 & 0.360 & 33.3 \\
\hline 40 & $3 D$ & 135 & 0.300 & 66.7 \\
\hline 60 & $3 D$ & 28 & 0.299 & 100 \\
\hline 20 & $4 D$ & 144 & 0.361 & 33.3 \\
\hline 40 & $4 D$ & 78 & 0.300 & 66.7 \\
\hline 60 & $4 D$ & 18 & 0.296 & 100 \\
\hline
\end{tabular}

Tabla 10: Número de pilotes y asentamiento total $\delta$ para $D=0.8$ $\mathrm{m}$ (pilote equivalente)

\begin{tabular}{|c|c|c|c|c|}
\hline$L, \mathrm{~m}$ & $S$ & pilotes & $\delta, \mathrm{m}$ & $L / D$ \\
\hline 20 & $2 D$ & 324 & 0.357 & 25 \\
\hline 40 & $2 D$ & 170 & 0.297 & 50 \\
\hline 60 & $2 D$ & 30 & 0.299 & 75 \\
\hline 20 & $3 D$ & 144 & 0.359 & 25 \\
\hline 40 & $3 D$ & 75 & 0.300 & 50 \\
\hline 60 & $3 D$ & 15 & 0.301 & 75 \\
\hline 20 & $4 D$ & 81 & 0.361 & 25 \\
\hline 40 & $4 D$ & 43 & 0.301 & 50 \\
\hline 60 & $4 D$ & 10 & 0.294 & 75 \\
\hline
\end{tabular}

Tabla 11: Número de pilotes y asentamiento total $\delta$ para $D=1.0$ $\mathrm{m}$ (pilote equivalente)

\begin{tabular}{|c|c|c|c|c|}
\hline$L, \mathrm{~m}$ & $S$ & pilotes & $\delta, \mathrm{m}$ & $L / D$ \\
\hline 20 & $2 D$ & 210 & 0.358 & 20 \\
\hline 40 & $2 D$ & 105 & 0.299 & 40 \\
\hline 60 & $2 D$ & 18 & 0.300 & 60 \\
\hline 20 & $3 D$ & 100 & 0.360 & 20 \\
\hline 40 & $3 D$ & 47 & 0.300 & 40 \\
\hline 60 & $3 D$ & 10 & 0.294 & 60 \\
\hline 20 & $4 D$ & 49 & 0.362 & 20 \\
\hline 40 & $4 D$ & 27 & 0.301 & 40 \\
\hline 60 & $4 D$ & 6 & 0.296 & 60 \\
\hline
\end{tabular}

\section{Método de Poulos y Davis}

Para el cálculo del asentamiento se parte del supuesto que la distribución de carga será de $70 \%$ pilotes y $30 \%$ placa, de acuerdo con lo establecido por Cooke et al. (1981) y Cooke (1986). La Tabla 12 muestra que, con la suposición de distribución de cargas, en ninguno de los casos se logró cumplir con el asentamiento de $30 \mathrm{~cm}$.

Con el fin de evaluar si esta metodología proporciona una solución frente al problema propuesto en esta investigación, se continuó aumentando el número de pilotes hasta que se cumpliera con el asentamiento de $30 \mathrm{~cm}$, superando el 100\% de la carga tomada, como se presenta en la Tabla 13.

Tabla 12: Asentamiento total $\delta$ y número de pilotes para una distribución de carga $70 \%$ pilotes y $30 \%$ placa

\begin{tabular}{|c|c|c|c|}
\hline$L, \mathrm{~m}$ & $D, \mathrm{~m}$ & pilotes & $\delta, \mathrm{m}$ \\
\hline 20 & 0.6 & 132 & 0.523 \\
\hline 40 & 0.6 & 69 & 0.479 \\
\hline 60 & 0.6 & 47 & 0.441 \\
\hline 20 & 0.8 & 100 & 0.529 \\
\hline 40 & 0.8 & 52 & 0.427 \\
\hline 60 & 0.8 & 35 & 0.412 \\
\hline 20 & 1.0 & 81 & 0.476 \\
\hline 40 & 1.0 & 41 & 0.411 \\
\hline 60 & 1.0 & 28 & 0.376 \\
\hline
\end{tabular}

Tabla 13: Asentamiento total $\delta$ y número de pilotes para un porcentaje de carga mayor o igual al $100 \%$

\begin{tabular}{|c|c|c|c|c|}
\hline$L, \mathrm{~m}$ & $D, \mathrm{~m}$ & pilotes & $\delta, \mathrm{m}$ & Carga, $\%$ \\
\hline 20 & 0.6 & 490 & 0.301 & 260 \\
\hline 40 & 0.6 & 180 & 0.298 & 182 \\
\hline 60 & 0.6 & 95 & 0.299 & 143 \\
\hline 20 & 0.8 & 423 & 0.299 & 253 \\
\hline 40 & 0.8 & 96 & 0.299 & 130 \\
\hline 60 & 0.8 & 60 & 0.296 & 121 \\
\hline 20 & 1.0 & 246 & 0.299 & 195 \\
\hline 40 & 1.0 & 70 & 0.300 & 118 \\
\hline 60 & 1.0 & 39 & 0.297 & 100 \\
\hline
\end{tabular}

\section{Método de Poulos-Davis-Randolph}

En las Tablas 14, 15 y 16, se resumen los resultados arrojados por la metodología de Poulos-Davis-Randolph. Con el propósito de mostrar la importancia de considerar el efecto que tiene el estrato blando que se encuentra por debajo de la cabeza de los pilotes, se muestran los asentamientos $\delta_{2}$ que se obtendrían al ignorar la presencia de dicha capa blanda y la diferencia $\Delta$ en $\%$ de los asentamientos. Se observa que para los pilotes de $20 \mathrm{~m}$ de longitud, los asentamientos sin considerar el efecto de la capa blanda son en promedio un 55\% menores respecto a los obtenidos teniendo en cuanta los asentamientos por consolidación de la capa blanda. Para el caso de los pilotes cuya longitud es de $40 \mathrm{~m}$, dicha diferencia es del $40 \%$ mientras que para los pilotes de mayor longitud, la diferencia es del $21 \%$. 
Tabla 14: Asentamiento total método PDR, $D=0.6 \mathrm{~m}$

\begin{tabular}{|c|c|c|c|c|}
\hline \multicolumn{5}{|c|}{$S=2 D$} \\
\hline Pilotes & $\delta_{1}, \mathrm{~m}$ & $\delta_{2}, \mathrm{~m}$ & $\Delta, \%$ & $L / D$ \\
\hline 625 & 0.732 & 0.334 & 54.4 & 33.3 \\
\hline 625 & 0.311 & 0.176 & 43.4 & 66.7 \\
\hline 100 & 0.305 & 0.243 & 20.3 & 100 \\
\hline \multicolumn{5}{|c|}{$S=3 D$} \\
\hline 256 & 0.726 & 0.32 & 55.9 & 33.3 \\
\hline 256 & 0.314 & 0.178 & 43.3 & 66.7 \\
\hline 60 & 0.298 & 0.237 & 20.5 & 100 \\
\hline \multicolumn{5}{|c|}{$S=4 D$} \\
\hline 144 & 0.716 & 0.311 & 56.6 & 33.3 \\
\hline 144 & 0.315 & 0.179 & 43.2 & 66.7 \\
\hline 40 & 0.298 & 0.238 & 20.1 & 100 \\
\hline
\end{tabular}

Tabla 15: Asentamiento total método PDR, $D=0.8 \mathrm{~m}$

\begin{tabular}{|c|c|c|c|c|}
\hline \multicolumn{5}{|c|}{$S=2 D$} \\
\hline Pilotes & $\delta_{1}, \mathrm{~m}$ & $\delta_{2}, \mathrm{~m}$ & $\Delta, \%$ & $L / D$ \\
\hline 324 & 0.756 & 0.351 & 53.6 & 25 \\
\hline 324 & 0.351 & 0.215 & 38.7 & 50 \\
\hline 78 & 0.298 & 0.237 & 20.5 & 75 \\
\hline \multicolumn{5}{|c|}{$S=3 D$} \\
\hline 144 & 0.34 & 0.34 & 54.4 & 25 \\
\hline 144 & 0.232 & 0.232 & 37 & 50 \\
\hline 42 & 0.24 & 0.24 & 20 & 75 \\
\hline \multicolumn{5}{|c|}{$S=4 D$} \\
\hline 81 & 0.337 & 0.337 & 53.4 & 25 \\
\hline 81 & 0.198 & 0.198 & 39.8 & 50 \\
\hline 30 & 0.236 & 0.236 & 20 & 75 \\
\hline
\end{tabular}

Tabla 16: Asentamiento total método PDR, $D=1.0 \mathrm{~m}$

\begin{tabular}{|c|c|c|c|c|}
\hline \multicolumn{5}{|c|}{$S=2 D$} \\
\hline Pilotes & $\delta_{1}, \mathrm{~m}$ & $\delta_{2}, \mathrm{~m}$ & $\Delta, \%$ & $L / D$ \\
\hline 225 & 0.746 & 0.348 & 53.4 & 20 \\
\hline 225 & 0.362 & 0.227 & 37.3 & 40 \\
\hline 70 & 0.299 & 0.239 & 20.1 & 60 \\
\hline \multicolumn{5}{|c|}{$S=3 D$} \\
\hline 100 & 0.743 & 0.345 & 53.6 & 20 \\
\hline 100 & 0.36 & 0.226 & 37.2 & 40 \\
\hline 38 & 0.297 & 0.239 & 24.3 & 60 \\
\hline \multicolumn{5}{|c|}{$S=4 D$} \\
\hline 49 & 0.773 & 0.362 & 53.2 & 20 \\
\hline 49 & 0.379 & 0.242 & 36.1 & 40 \\
\hline 25 & 0.299 & 0.241 & 19.4 & 60 \\
\hline
\end{tabular}

Teniendo en cuenta el criterio de los $30 \mathrm{~cm}$ de asentamiento máximo, el $70.4 \%$ de soluciones obtenidas sin considerar la capa blanda cumplirían dicha condición y cuentan con la posibilidad de optimizar el número de pilotes, dado que los asentamientos calculados se encuentran muy por debajo de los $30 \mathrm{~cm}$. Sin embargo, si se analiza el caso contrario, únicamente el 33.3\% (solo los pilotes de $60 \mathrm{~m}$ de longitud) de las soluciones sería adecuada para cumplir lo establecido en la norma. Por lo que queda en evidencia la desventaja que posee esta metodología para el diseño de sistemas placa-pilote en la ciudad de Bogotá, en especial cuando quienes realizan los diseños de dichos sistemas no tienen conocimiento de todas las consideraciones adicionales a la metodología original propuesta en 1994.

\section{Método de Elementos Finitos 3D FEM3D}

Los elementos finitos permiten evaluar la influencia de un problema no lineal, con la incorporación de un modelo más avanzado que admite representar la tridimensionalidad de un problema placa-pilote. Diferentes análisis de sistemas placa-pilote han sido desarrollados con la ayuda de elementos finitos 3D (Hansbo y Jendeby, 1983; Katzenbach et al., 1998; Reul y Randolph, 2003), quienes buscaron determinar la influencia de la configuración geométrica de los elementos (placa y pilotes) en el cálculo de asentamientos, representando la variación del asentamiento en función del cambio de espesor de placa, separación, longitud y diámetro de los elementos profundos. Con el fin de determinar el impacto que una $u$ otra propiedad geométrica causa en la estimación de los asentamientos, se representaron gráficamente las combinaciones mencionadas desarrolladas en esta investigación.

La representación de los asentamientos en sistemas placapilote para este caso fue realizada bajo el criterio de una placa flexible y rígida, donde se estimaron las diferencias bajo ambas condiciones de análisis, variando el espesor de placa, diámetro, longitud y separación entre pilas para cada una de las combinaciones. La variación del asentamiento en post-construcción fue estimada como la diferencia entre el desplazamiento promedio a 20 años menos el desplazamiento promedio al finalizar construcción. En las Figuras 10 y 11, se muestra la variación de los asentamientos en función del diámetro. Se encontró que la variación del diámetro representó para los sistemas placa 
pilote un aumento promedio en los asentamientos del $4.6 \%$ al aumentar de diámetro $0.6 \mathrm{~m}$ a $0.8 \mathrm{~m}, 18.5 \%$ al aumentar de $0.8 \mathrm{~m}$ a $1.0 \mathrm{~m}$ y del $26.6 \%$ al aumentar el diámetro de 0.6 $\mathrm{m}$ a $1.0 \mathrm{~m}$. Esto muestra que el efecto que tiene el aumento de diámetro (y por consiguiente la reducción del número de pilotes dado que es menor el número de pilotes que pueden ser construidos dentro del área de placa) es mucho menor cuando el cambio de diámetro es de $0.6 \mathrm{~m}$ a $0.8 \mathrm{~m}$ por lo que se puede concluir que para las condiciones geotécnicas y carga aplicada en esta investigación los diámetros que mayor eficiencia muestran son los de $0.6 \mathrm{~m}$ y $0.8 \mathrm{~m}$.

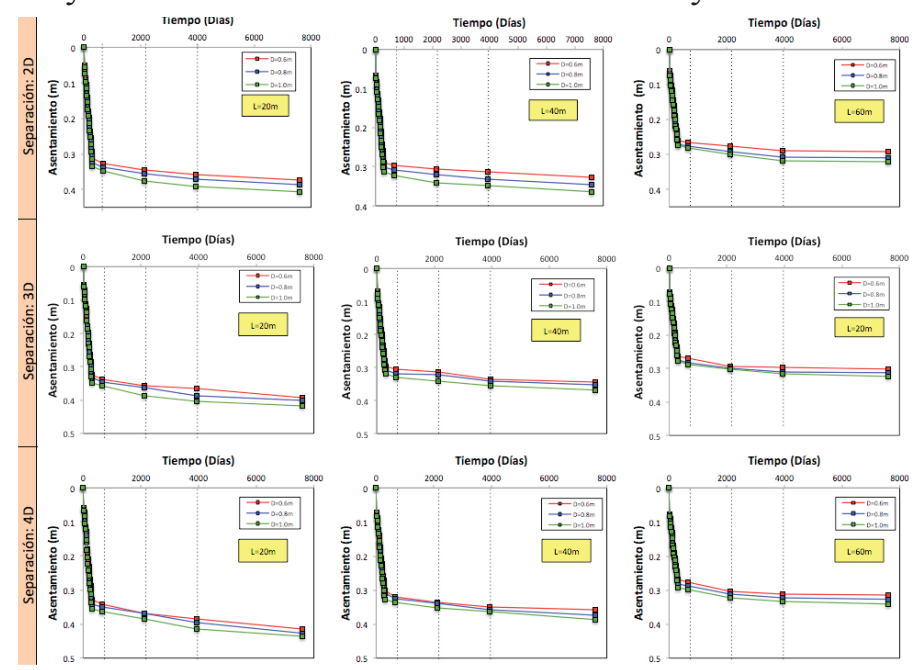

Figura 10: Efecto del diámetro en los asentamientos totales para placa con espesor de $0.5 \mathrm{~m}$

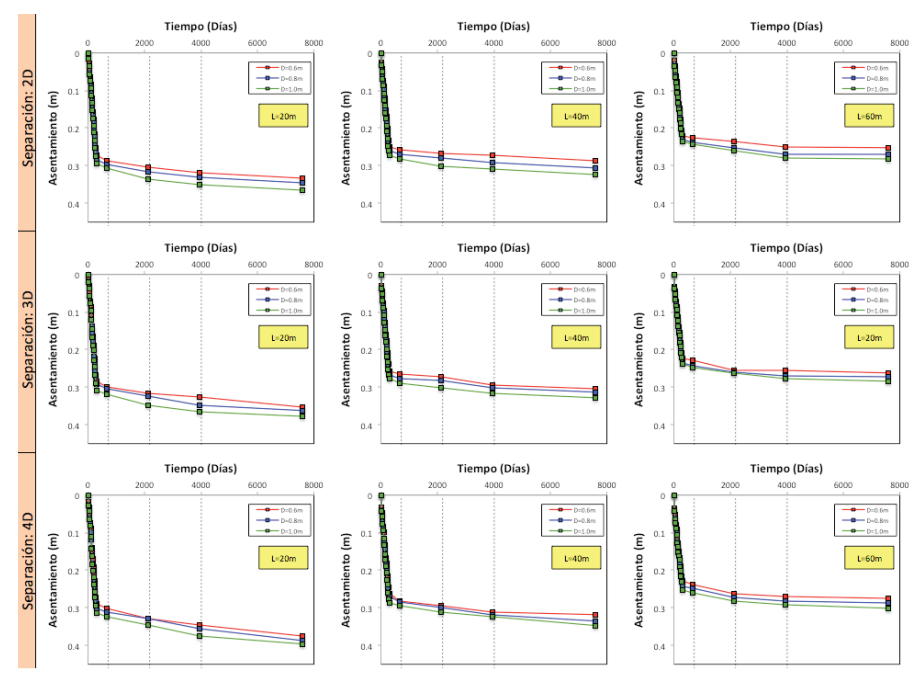

Figura 11: Efecto del diámetro en los asentamientos totales para placa con espesor de $1.0 \mathrm{~m}$

Con el fin de seguir evaluando las variables geométricas que mayor incidencia tiene en la variación de los asentamientos, se graficó la variación de los mismos (para cada espesor de placa) respecto a la longitud de los pilotes. En las Figuras 12 y 13, se puede apreciar que, al aumentar la longitud de los pilotes, se reduce los asentamientos entre el 41.2 y $46.6 \%$ con un valor promedio de $44 \%$ para cuando la longitud de pilotes se aumenta de 20 a $40 \mathrm{~m}$ respectivamente. En el caso cuando la longitud de los pilotes se aumenta de 40 a $60 \mathrm{~m}$, la reducción que se estimó fue entre 15.4 y $16.9 \%$ con un valor promedio de $16 \%$. Por lo tanto, se puede concluir que la longitud de los pilotes representa la variable geométrica con mayor importancia para la reducción de asentamientos. Sin embargo, es posible que un aumento de longitud de pilotes mayores a $60 \mathrm{~m}$ no genere una disminución significativa en el desplazamiento vertical promedio del sistema placapilote con la misma eficiencia que los registrados para pilotes con longitudes de 20 a $40 \mathrm{~m}$.

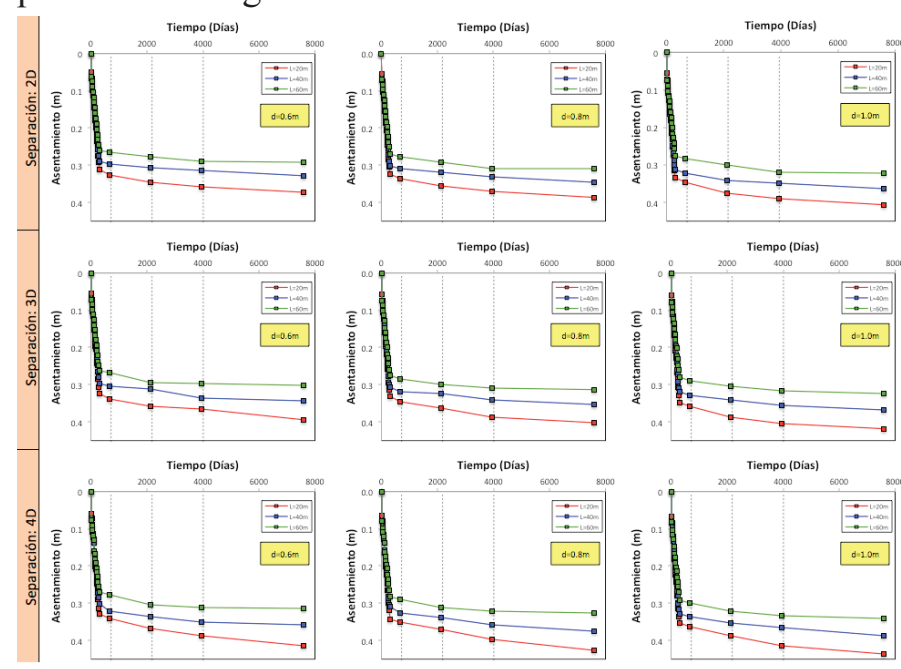

Figura 12: Efecto de la longitud en los asentamientos totales para placa con espesor de $0.5 \mathrm{~m}$

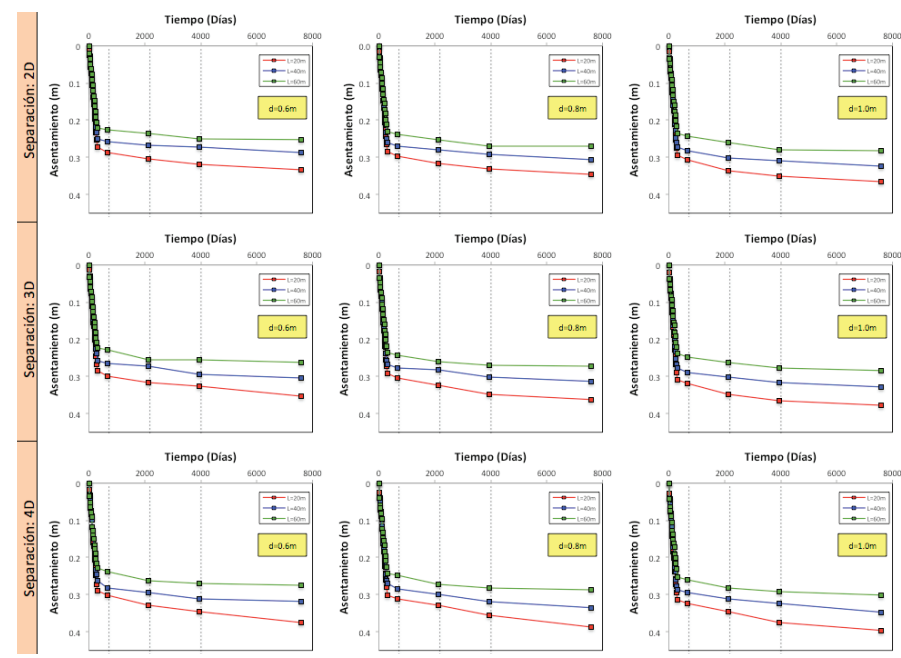

Figura 13. Efecto de la longitud en los asentamientos totales para placa con espesor de $1.0 \mathrm{~m}$ 
De las Figuras 14 y 15, se observa que a medida que se disminuyó la separación entre pilotes, fue posible reducir los asentamientos entre $14.3 \%$ y $19.2 \%$. Esto se debe a que cuando las separaciones son menores, el número de pilotes que pueden ser construidos en el área de placa aumenta por lo que en teoría el control de asentamientos se puede realizar de mejor manera. Aunque el objetivo de los pilotes en los sistemas placa pilote no es principalmente lograr una reducción (como grupo) de los esfuerzos inducidos por la carga aplicada, sí se evidenció que los menores asentamientos se obtuvieron cuando el diámetro de los pilotes era pequeño, la longitud superior a $40 \mathrm{~m}$ y la separación era dos veces el diámetro.

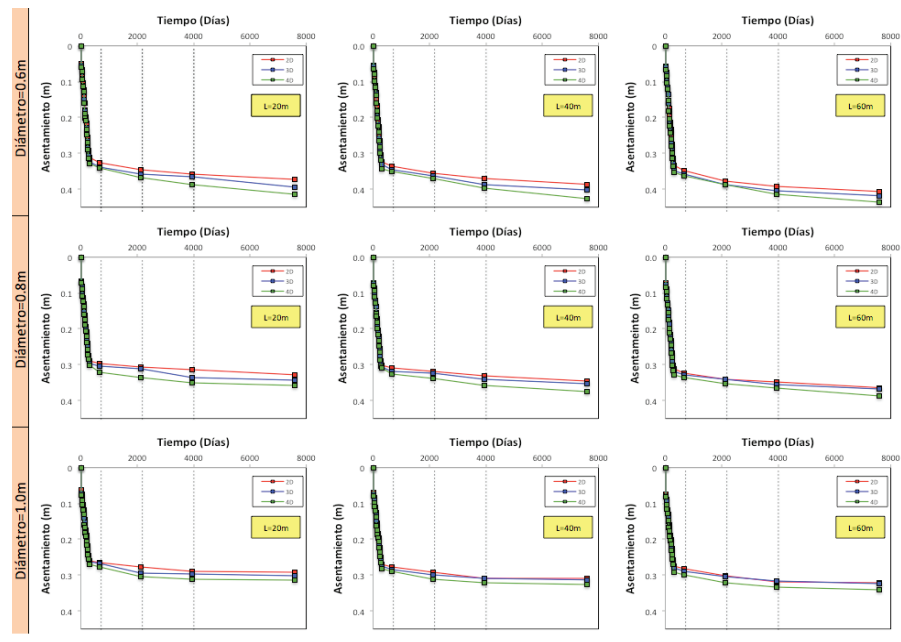

Figura 14: Efecto de la separación en los asentamientos totales para placa con espesor de $0.5 \mathrm{~m}$

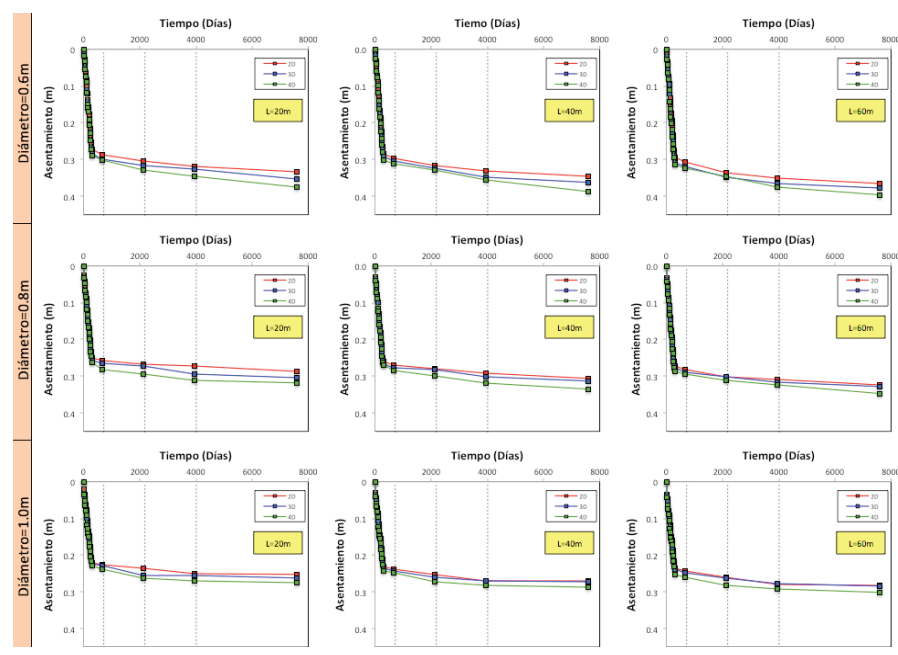

Figura 15: Efecto de la separación en los asentamientos totales para placa con espesor de $1.0 \mathrm{~m}$

Igualmente se representó la variabilidad de los asentamientos durante las etapas constructivas frente al espesor de la placa (flexible y rígida), diámetro, longitud y separación entre elementos profundos, con el fin de observar la variación del asentamiento promedio en el centro, teniendo en cuenta el proceso de construcción de la carga por etapas e instantáneamente. En las Figuras 16 a 19, la línea SPC se refiere a la estimación del asentamiento sin tener en cuenta el proceso constructivo para la configuración $D=0.6 \mathrm{~m}, L=20$ m y $S=2 D$. Las demás líneas representan por franjas de color, rojo $(D=0.6 \mathrm{~m})$, azul $(D=0.8 \mathrm{~m})$ y verde $(D=1.0 \mathrm{~m})$, la variación del asentamiento total desde el primer día de aplicación de la carga, hasta finalizar la construcción (300 días).

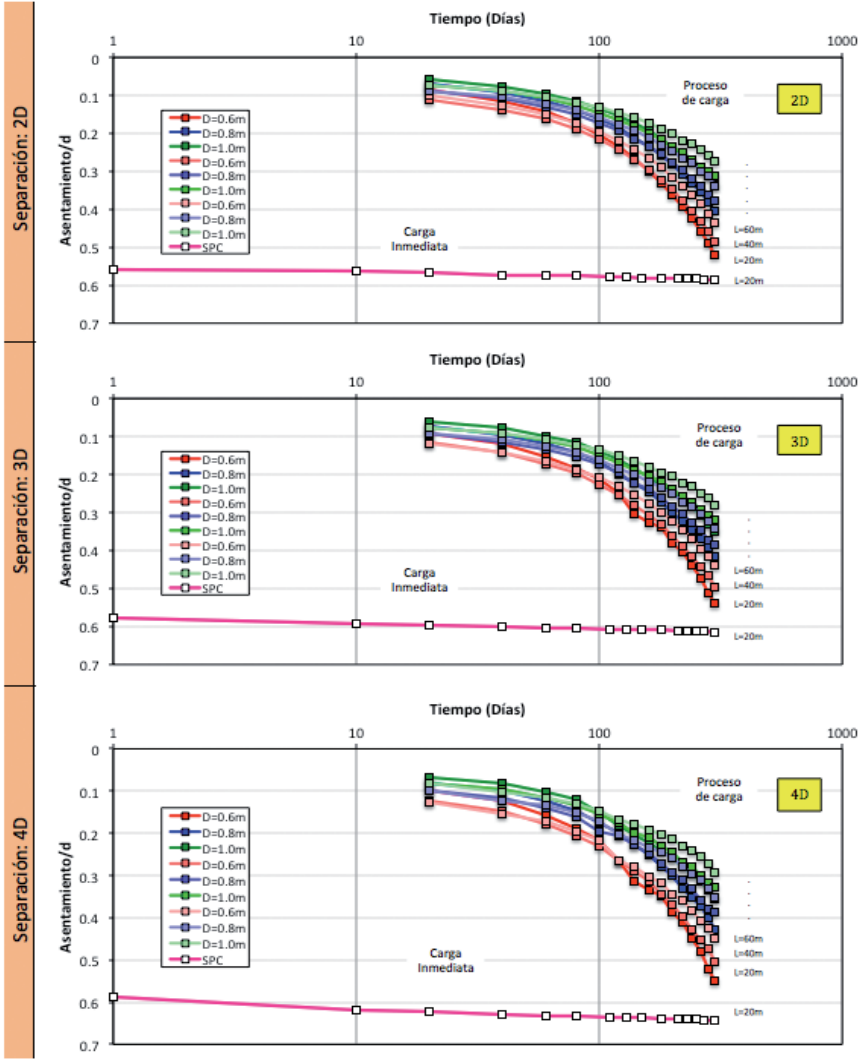

Figura 16: Efecto del diámetro en el comportamiento de los asentamientos totales durante y post-construcción para placa con espesor de $0.5 \mathrm{~m}$

En primera instancia pudo identificarse que, durante el proceso de carga, la propiedad geométrica que mayor incidencia tiene en la variación del asentamiento es la longitud, logrando una reducción del asentamiento en tantas veces la longitud sea mayor. Asimismo, se confirmó que la variación de longitudes de 20 ó $60 \mathrm{~m}$ presenta un rango amplio en el cual fue posible reducir el asentamiento hasta en un $75 \%$ con el aumento de la longitud. 


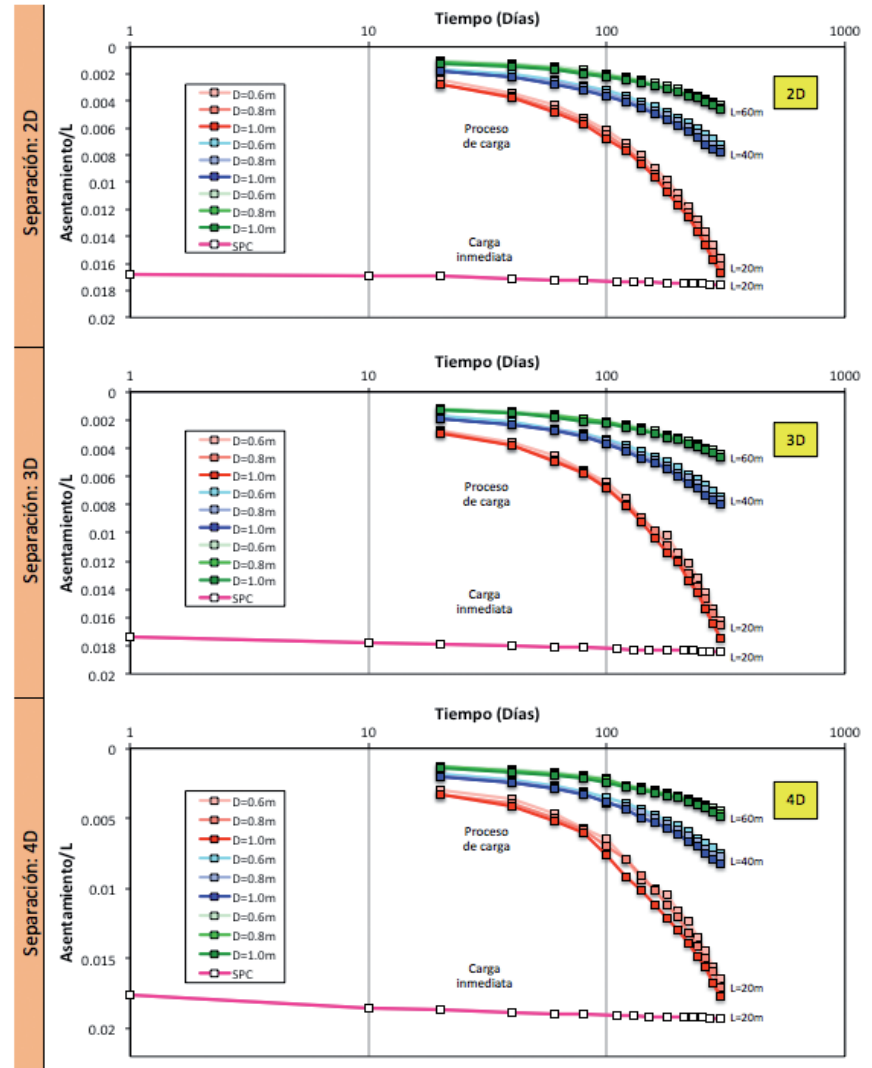

Figura 17: Efecto de la longitud en el comportamiento de los asentamientos totales durante y post-construcción para placa con espesor de $0.5 \mathrm{~m}$

En caso contrario, la variación de longitudes de 40 ó $60 \mathrm{~m}$ presentó una reducción en el asentamiento del $41 \%$. Para alcanzar a lograr que el diámetro tenga una incidencia similar a la longitud en la estimación de los asentamientos de sistemas placa pilote, sería necesario que esta aumentará en tantas veces que su dimensión fuera al menos lo suficientemente superior al valor del asentamiento obtenido para los diámetros convencionales propuestos. Sin embargo, se presenta la dificultad para la construcción de pilas con diámetros superiores a 1.0 $\mathrm{m}$ por la disponibilidad de equipo y maquinaria. Bajo el proceso de aplicación de la carga instantánea y por etapas, se evidenció que el valor de asentamiento hasta el punto de finalización de construcción (300 días) varía entre un 10.4 a $12.8 \%$ más, lo que significa que representar la estimación de asentamientos para un problema de cimentación combinada (placa + pilotes), sin tener en cuenta el avance de construcción en la obra puede sobre estimar los valores de asentamiento.

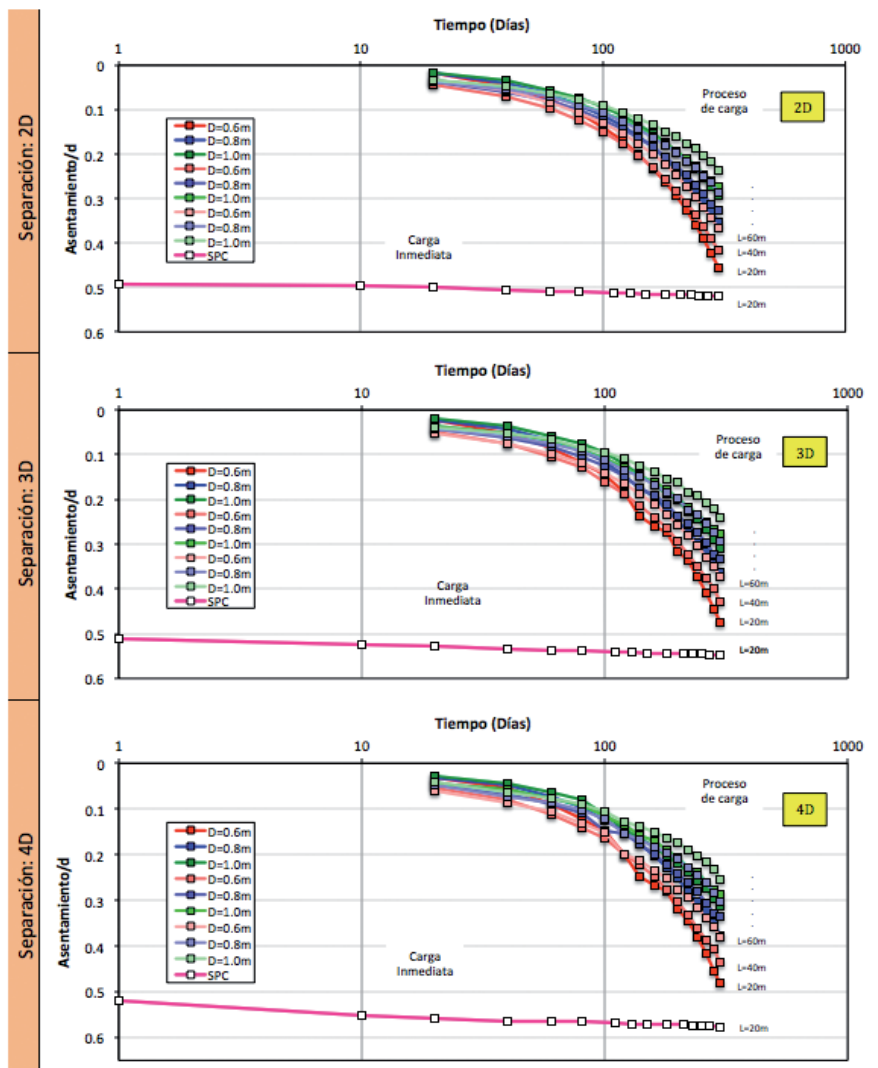

Figura 18: Efecto del diámetro en el comportamiento de los asentamientos totales durante y post-construcción para placa con espesor de $1.0 \mathrm{~m}$

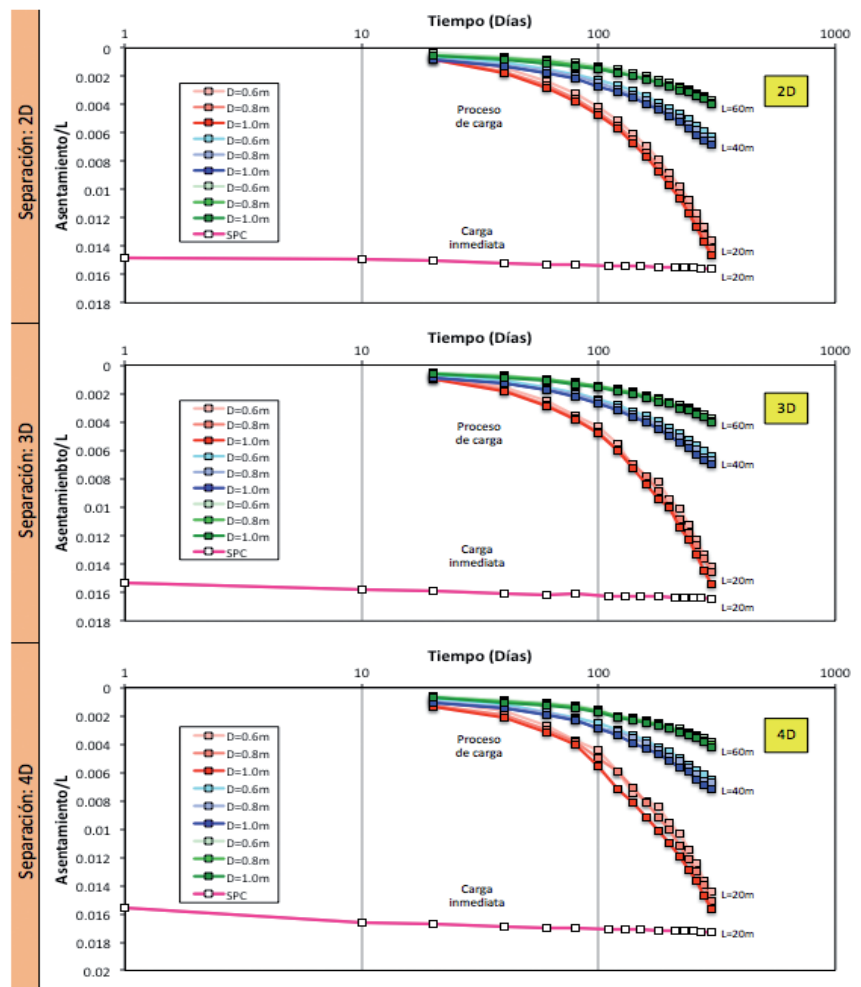

Figura 19: Efecto de la longitud en el comportamiento de los asentamientos totales durante y post-construcción para placa con espesor de $1.0 \mathrm{~m}$ 
Para este caso de estudio, el hecho de evaluar un modelo por etapas, permitió que los excesos de presión de poros tuvieran un tiempo de disiparse entre cada construcción por piso (20 días), mientras que la evaluación simplificada de aplicación de carga instantánea, reflejó una elevada estimación de los excesos de presión de poros, muestra de esto fueron las diferencias en el asentamiento total al analizar la construcción. Se concluye que evaluar los asentamientos totales teniendo en cuenta las etapas de construcción, permite optimizar el sistema de cimentación y lograr un menor error en la predicción del desplazamiento vertical del sistema combinado.

\section{Asentamientos diferenciales}

Con el fin de ver el efecto que tienen los pilotes sobre los asentamientos diferenciales del sistema en cada uno de los modelos de elementos finitos, se graficó el perfil de asentamientos longitudinal (desde el borde del modelo hasta el eje de simetría), tal como se muestra en la Figura 20.

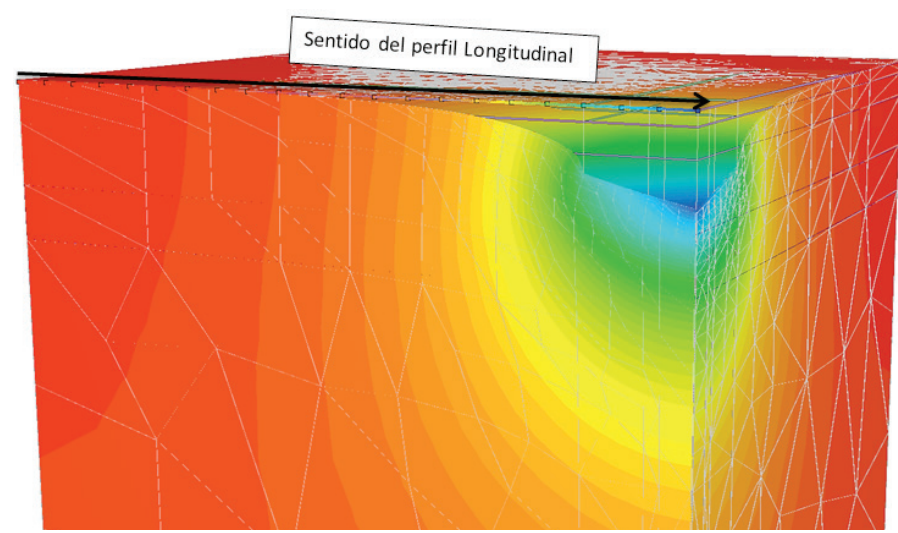

Figura 20: Orientación del perfil longitudinal para la determinación de asentamientos diferenciales

A continuación, las Figuras 21 a 24 presentan los tipos de comportamiento generales observados para cada una de las configuraciones.

Se observa que, para condiciones idealizadas de carga uniforme, el perfil de asentamientos de la cimentación formada únicamente por la placa tiene forma de una "artesa", donde los asentamientos son de mayor magnitud en el centro y menores en el borde. Al introducir los pilotes (cuya función es la reducción de asentamientos) dicha forma se ve afectada dependiendo de si la distribución del número de pilotes se realiza en toda la placa o si se concentra en el centro de la misma. Esto concuerda con lo reportado por Tan et al. (2005). Bajad y Sahu (2009) encontraron que la adición de pilotes conlleva a la reducción de los asentamientos diferenciales en un rango del 40 al $60 \%$ para los sistemas placa-pilote con distribución de pilotes por toda la placa y 40 a $70 \%$ para sistemas placapilote con los pilotes ubicados en el centro de la placa. Finalmente, Bajad y Sahu (2009) concluyen que un grupo de pilotes distribuido en la porción central de alrededor del 20 al 40\% del área de la placa, logra ser más efectivo en la reducción de los asentamientos diferenciales. Randolph (1994) reportó que para sistemas placa-pilote cargados de manera uniforme, los pilotes deben concentrarse en el 15 a $25 \%$ del área de la placa.

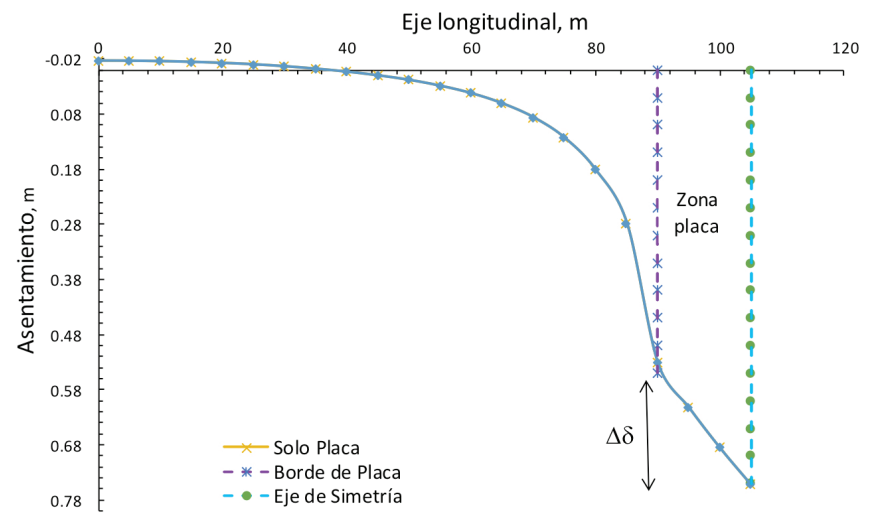

Figura 21: Perfil longitudinal de asentamientos diferenciales para un modelo sin pilotes y placa con espesor de $0.5 \mathrm{~m}$. Configuración solo placa y $\Delta \delta=0.22 \mathrm{~m}$

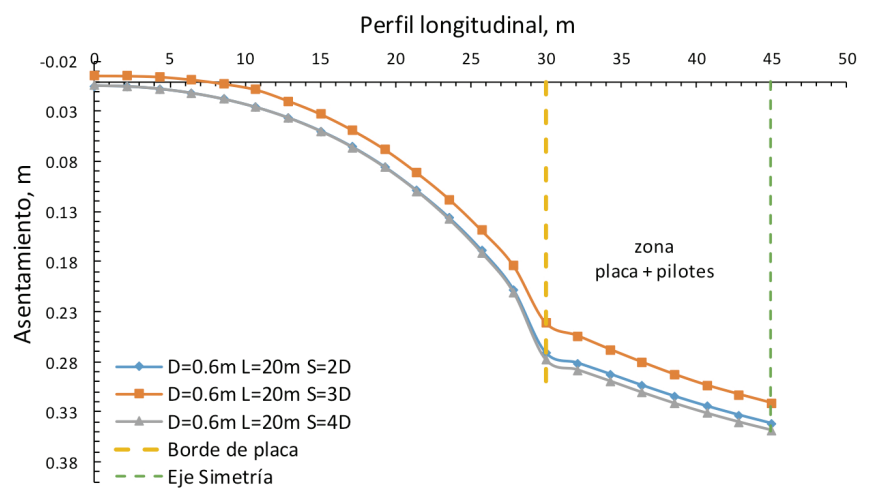

Figura 22: Perfil longitudinal de asentamientos diferenciales para un modelo $D=0.6 \mathrm{~m}, L=20 \mathrm{~m}, S=2 D(399$ pilotes, $\Delta \delta=$ $0.07 \mathrm{~m}), 3 D(225$ pilotes, $\Delta \delta=0.08 \mathrm{~m})$ y $4 D$ (121 pilotes, $\Delta \delta=$ $0.07 \mathrm{~m})$

Se encontró que cuando la separación de los pilotes es igual a 2 veces su diámetro y el área de ocupación de los pilotes ocupa en promedio el 37\% de la placa, la reducción de los asentamientos alcanza su máximo valor (81\%). A su vez, para cuando la separación es de 3 veces el diámetro 
y el área promedio de ocupación en planta ocupada por los pilotes es del $59 \%$, es cuando se logra una reducción promedio del $91 \%$. Por lo que se puede concluir que la separación recomendada para lograr la mayor reducción en los asentamientos diferenciales, teniendo en cuenta las condiciones geotécnicas y geométricas de esta investigación es 3 veces el diámetro.

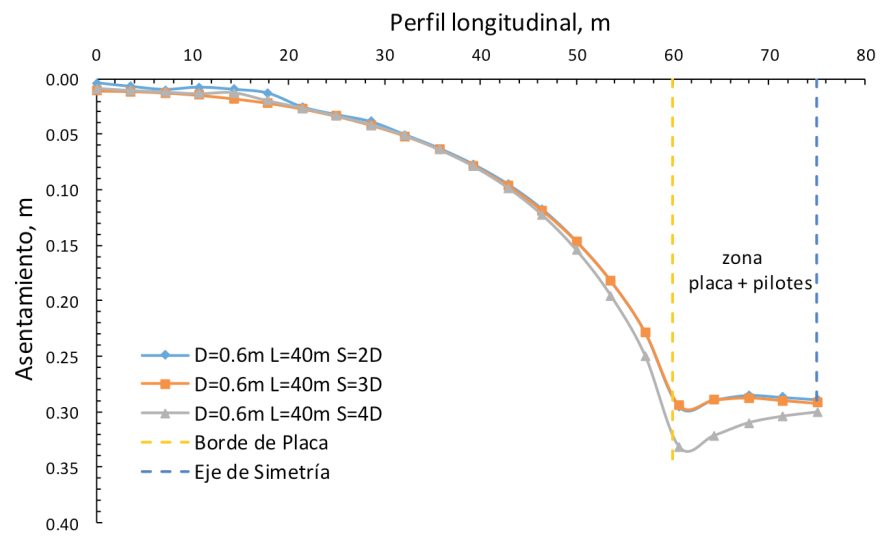

Figura 23: Perfil longitudinal de asentamientos diferenciales para un modelo $D=0.6 \mathrm{~m}, L=40 \mathrm{~m}, \mathrm{~S}=2 D(225$ pilotes, $\Delta \delta=$ $0.007 \mathrm{~m}), 3 D(121$ pilotes, $\Delta \delta=0.002 \mathrm{~m})$ y $4 D(49$ pilotes, $\Delta \delta=$ $0.032 \mathrm{~m})$

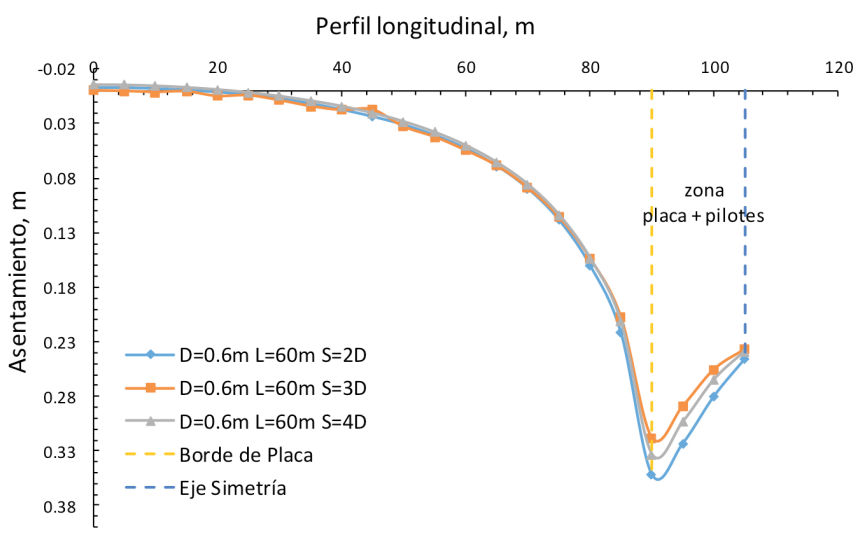

Figura 24: Perfil longitudinal de asentamientos diferenciales para un modelo $D=0.6 \mathrm{~m}, L=60 \mathrm{~m}, \mathrm{~S}=2 D(49$ pilotes, $\Delta \delta=$ $0.106 \mathrm{~m}), 3 D(49$ pilotes, $\Delta \delta=0.081 \mathrm{~m})$ y $4 D(25$ pilotes, $\Delta \delta=$ $0.095 \mathrm{~m})$

Comparando los resultados obtenidos en esta investigación con los reportados por los diferentes autores, se logra ser más efectivo en la reducción de los asentamientos diferenciales cuando los pilotes se concentran en el 37 a 59\% del área de la placa para sistemas placa-pilote cargados de manera uniforme. El comportamiento observado en la Figura 24, corresponde a los casos donde el área ocupada por el grupo de pilotes está por debajo del $30 \%$ del área de la placa. $\mathrm{Si}$ bien se logran reducir los asentamientos diferenciales en gran medida, por la forma en la que se deforma el sistema, es necesario tomar medidas especiales en el refuerzo del área que se encuentre por fuera de la zona donde se concentran los pilotes, lo que causa un incremento en los costos de construcción.

\section{Distribución de carga entre placa y pilotes}

Para la determinación de la distribución de cargas entre la placa y los pilotes obtenidos en cada una de las simulaciones se sumaron las cargas axiales tomadas por cada uno de los pilotes para luego obtener el total de la carga tomada por el grupo de pilotes. En las Figuras 25 y 26 se muestran los resultados obtenidos.

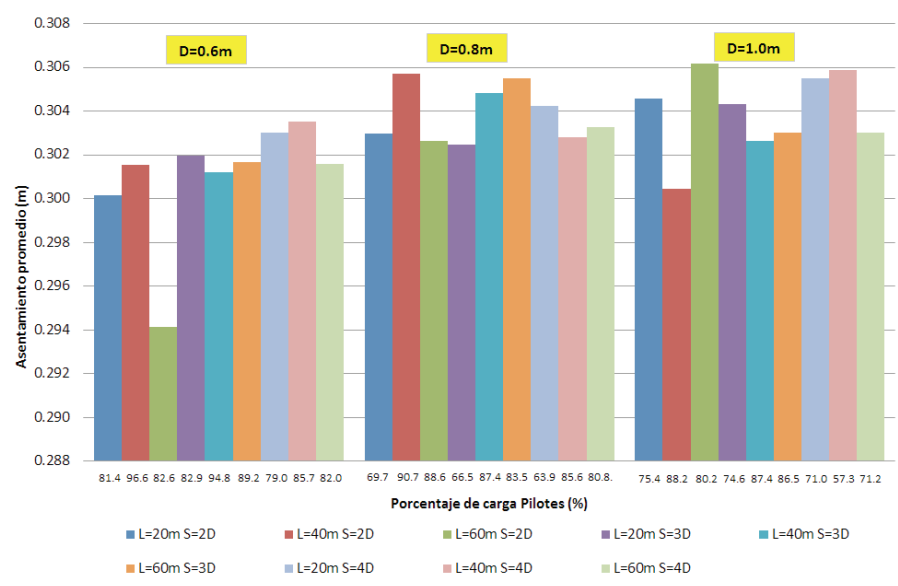

Figura 25: Relación de asentamientos totales y distribución de cargas para placa con espesor de $0.5 \mathrm{~m}$

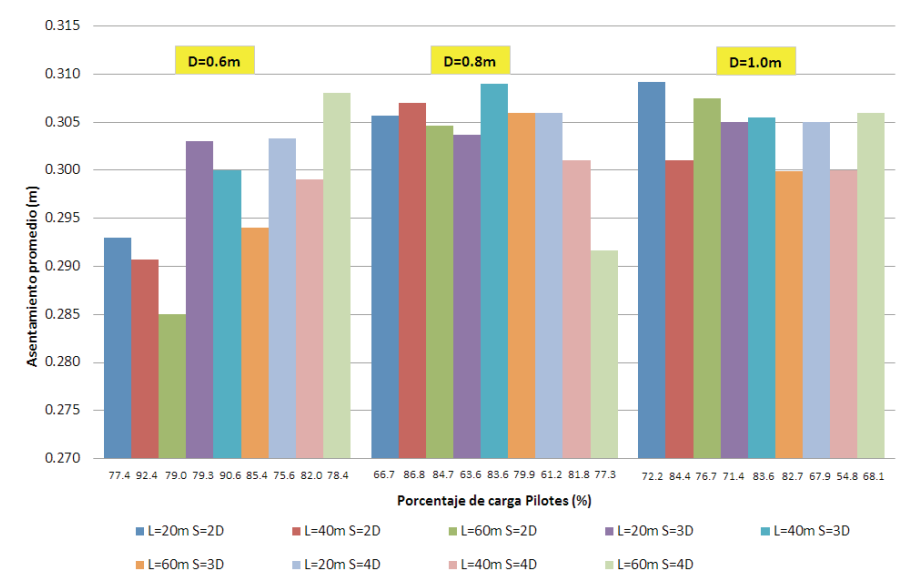

Figura 26: Relación de asentamientos totales y distribución de cargas para placa con espesor de $1.0 \mathrm{~m}$

Davids et al. (2008) concluyeron que las cargas de una superestructura son compartidas entre la placa y los pilotes, con los pilotes tomando entre 50 y $80 \%$ de la totalidad de la carga. Leung et al. (2010) observaron que el porcentaje de carga tomado por la placa en un sistema placa pilote 
varía entre el $25 \%$ al $51 \%$ de la totalidad de carga aplicada con un valor promedio de $36 \%$ cuando se diseña como una cimentación placa-pilote. Elwakil y Azzam (2016) reportaron parte de la carga de la superestructura que pasa al suelo a través del contacto con la placa varía entre 30 y $60 \%$ y depende del estado del suelo debajo de la superficie.

Esta investigación arrojó que, para los casos de placas con espesores de $50 \mathrm{~cm}$, el porcentaje de carga tomado por los pilotes varía ente el 57 y $97 \%$ con un valor promedio de $81 \%$ y que para el caso donde la placa tiene un espesor de $1.0 \mathrm{~m}$ el porcentaje de carga varía en un rango de 55 y $91 \%$ con valor promedio de $77 \%$. Se puede observar que el espesor de placa sí influye en el porcentaje de carga que toman los pilotes, siendo mayor cuando la placa tiene un espesor de $50 \mathrm{~cm}$. Sin embargo, dicha variación es únicamente del $4 \%$ por lo que se puede concluir que para el caso de análisis propuesto en esta investigación la placa de $50 \mathrm{~cm}$ se comporta como una placa rígida y no una flexible como se supuso inicialmente.

\section{Exceso de presión de poros}

En las Figuras 27, 28 y 29, se resume el comportamiento numérico de los excesos de presiones de poros, teniendo en cuenta la distribución de pilotes respecto al área de la placa y la longitud.

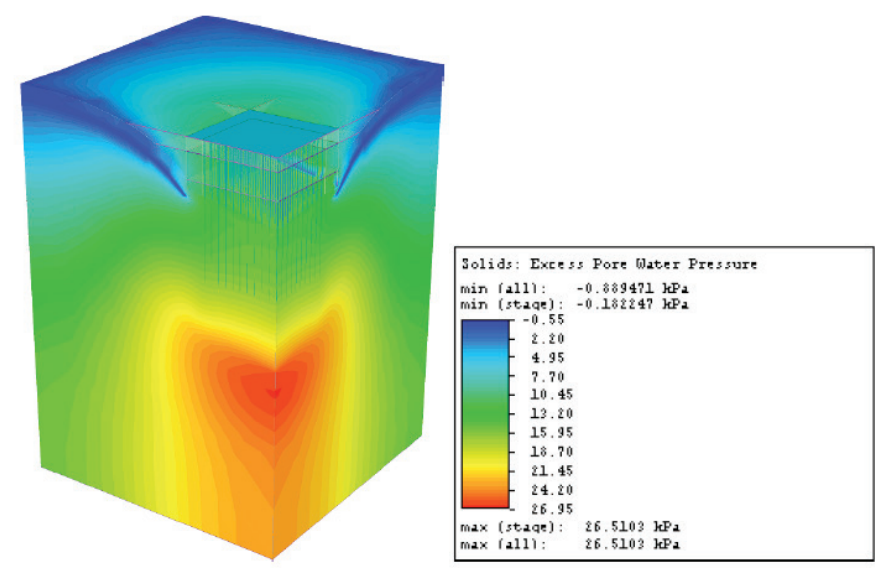

Figura 27: Modelo FEM, resultados de excesos de presiones de poros, configuración $D=0.6 \mathrm{~m}, L=20 \mathrm{~m}, S=2 D$ finalizada la construcción

Se puede observar que los mayores excesos de presiones de poros se manifiestan en la punta de los pilotes y que dicho valor va disminuyendo a medida que se aumenta la longitud de los pilotes. Se presenta la mayor reducción al pasar de $20 \mathrm{~m}$ a $40 \mathrm{~m}$ de longitud teniendo un $46 \%$ en promedio, mientras que la reducción en el valor de los excesos de presiones de poros para longitudes entre $40 \mathrm{~m}$ y $60 \mathrm{~m}$ es de únicamente del $10 \%$.
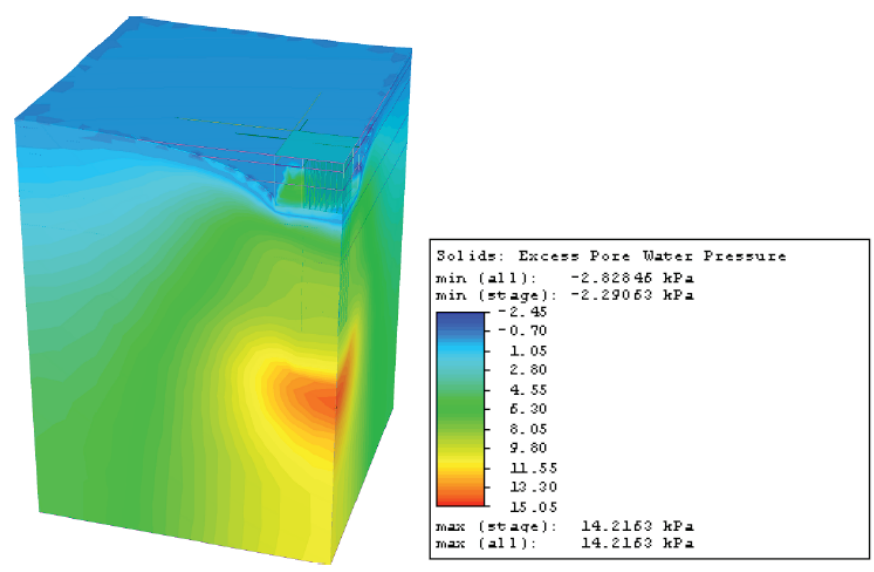

Figura 28: Modelo FEM, resultados de excesos de presiones de poros, configuración $D=0.6 \mathrm{~m}, L=40 \mathrm{~m}, \mathrm{~S}=2 D$ finalizada la construcción

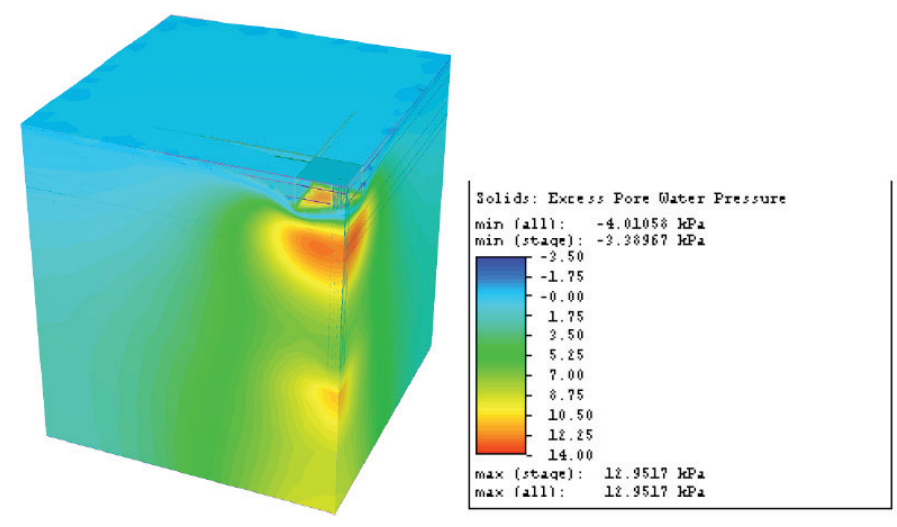

Figura 29: Modelo FEM, resultados de excesos de presiones de poros, configuración $D=0.6 \mathrm{~m}, L=60 \mathrm{~m}, \mathrm{~S}=2 \mathrm{D}$ finalizada la construcción

Adicionalmente se observa que en las configuraciones donde los pilotes se concentran en el centro de la placa (menor al 30\% del área de la placa) se presentan excesos de presiones de poros tanto debajo de la placa como en la punta de los pilotes, siendo mayores los excesos que se presentan debajo de la placa. Esto podría explicar el comportamiento observado en los asentamientos diferenciales debido a la diferencia entre dichos excesos de presiones y su tiempo de disipación. Respecto al efecto que tiene la separación de los pilotes en los excesos de presiones de poros, se observó que el incremento de excesos de presiones de poros aumenta entre 3 y $5 \%$ a medida que aumenta la separación entre centros de elementos verticales. 


\section{Comparación entre metodologías}

Finalizado el cálculo de los asentamientos totales, queda en evidencia la variabilidad que existe entre metodologías, en lo que respecta a la solución necesaria para cumplir con los asentamientos admisibles propuestos por la NSR-10 (2010). Los gráficos de barras de la Figura 30 muestran las variaciones de los asentamientos.

De la Figura 30 se puede concluir que el método PDR no arrojó ninguna solución que lograra cumplir con un

a)

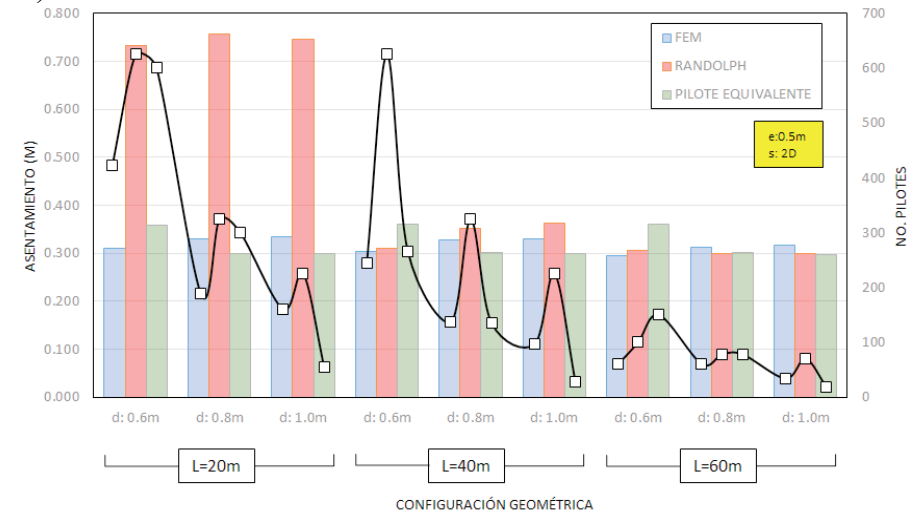

b)

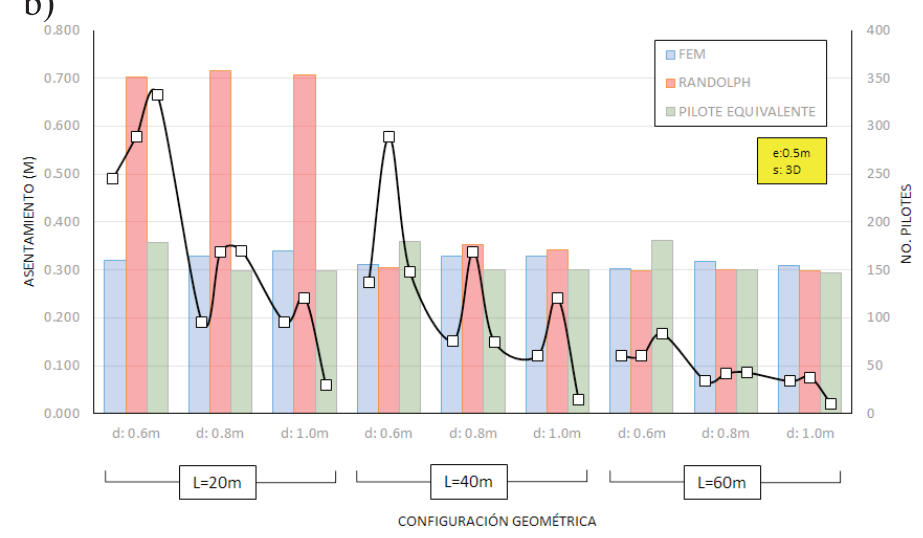

c)

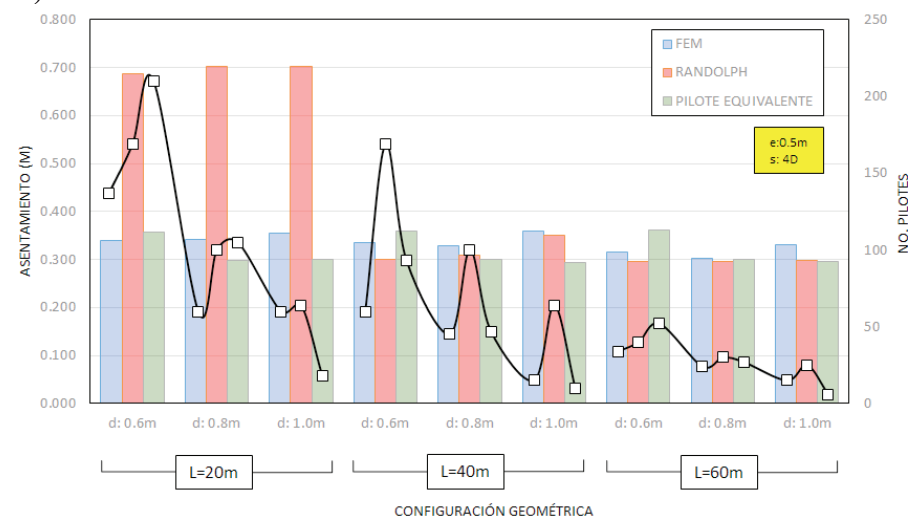

asentamiento de $30 \mathrm{~cm}$ para pilotes de diámetro $0.6 \mathrm{~m}$ y longitud de $20 \mathrm{~m}$, condición que posiblemente esté relacionada con la longitud de los pilotes. Al ser los pilotes de menor longitud, el espesor de suelo que se incluye en los cálculos de los asentamientos por consolidación, es mayor en comparación a los de pilotes de mayor longitud. Adicionalmente, la disipación de carga que proporciona dicho pilote es mucho menor dado que depende del factor $r_{\mathrm{m}}$ (radio de influencia del pilote), el cual depende a su vez de la longitud del pilote, por lo que el incremento de

d)

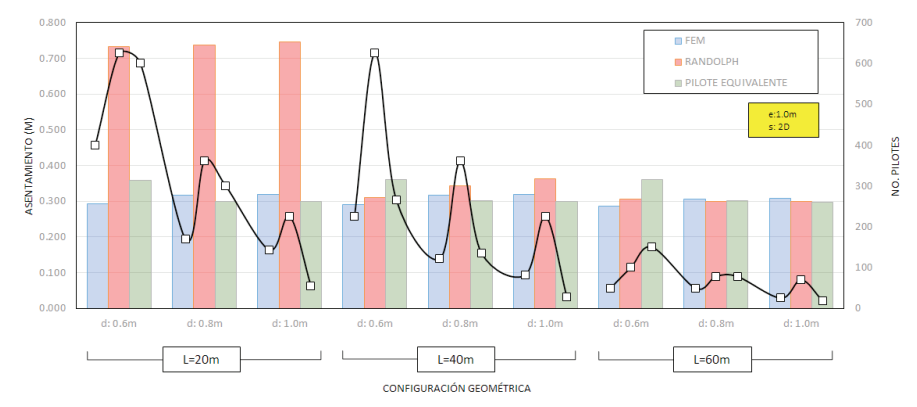

e)

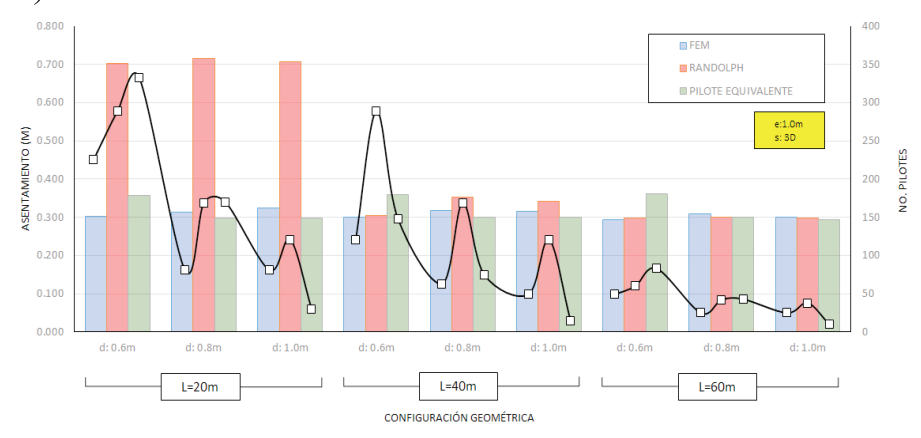

f)

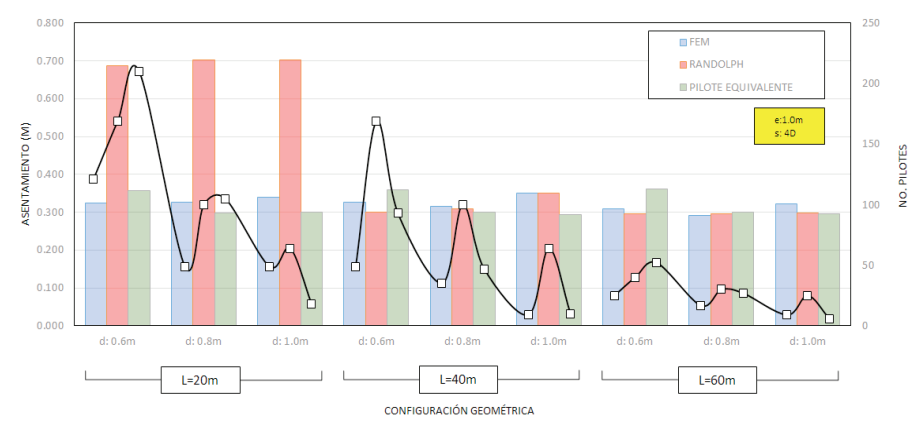

Figura 30: Variación de asentamientos totales entre metodologías para espesor de placa de: a) $0.5 \mathrm{~m}$ y $S=2 D$, b) $0.5 \mathrm{~m}$ y $S=3 D$, c) $0.5 \mathrm{~m}$ y $S=4 D$, d) $1 \mathrm{~m}$ y $S=2 D$, e) $1 \mathrm{~m}$ y $S=3 D$ y f) $1 \mathrm{~m}$ y $S=4 D$ 
esfuerzos que experimentan los estratos subyacentes a la punta de los pilotes va a influenciar los desplazamientos del suelo. Para los pilotes de mayor longitud de 40 a $60 \mathrm{~m}$, la diferencia que existe entre los asentamientos calculados por FEM y el método de Randolph varía entre 3 y $13 \%$ con un promedio del $6 \%$. Si se realiza la misma comparación entre FEM y el método del pilote equivalente, la diferencia varía entre 2 y $27 \%$ con un promedio del $10 \%$. Por lo tanto, puede concluirse que para elementos verticales con una longitud menor o igual a $20 \mathrm{~m}$, el método simplificado más conveniente y confiable es el de pilote equivalente, mientras que para pilotes con una longitud mayor, los métodos

a)

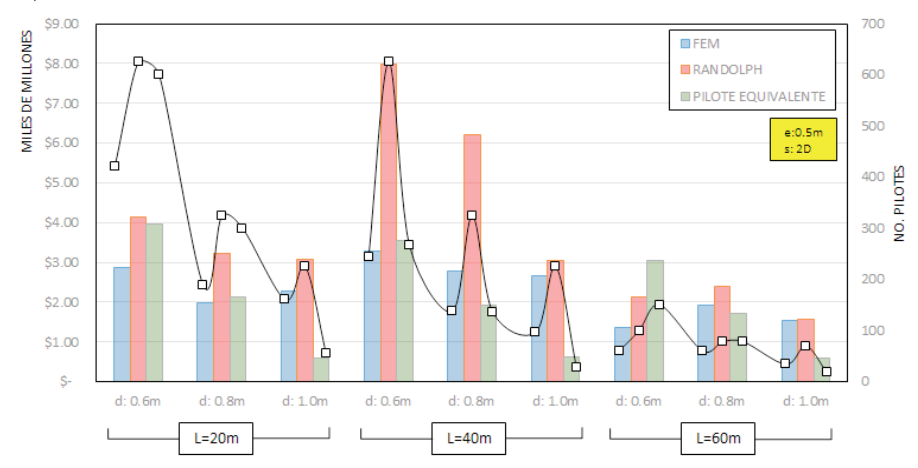

b)

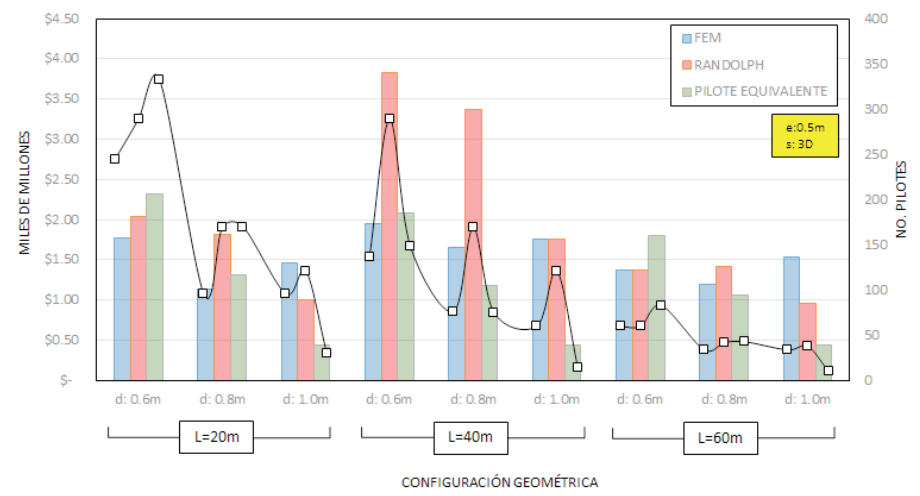

c)

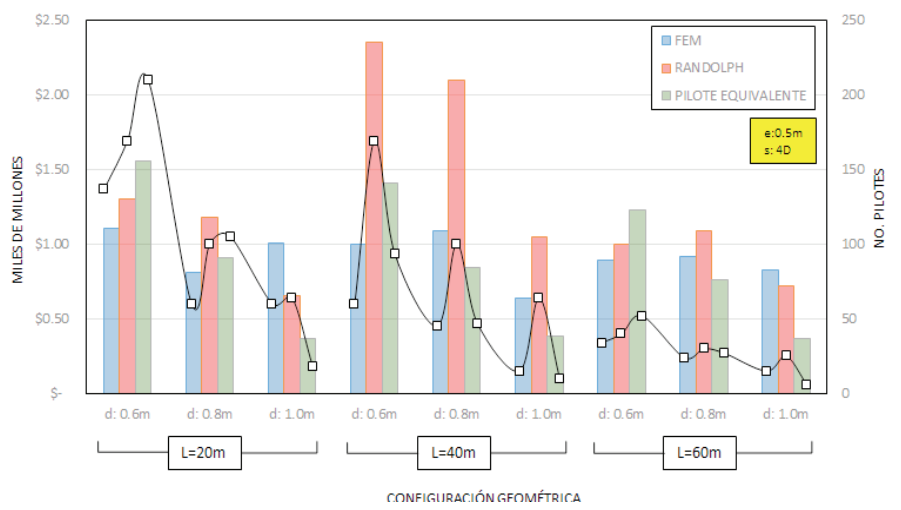

simplificados más confiables son pilote equivalente y PDR.

\section{Costos globales}

De acuerdo al cálculo de precios globales de construcción, tomado como referencia por $\mathrm{m}^{3}$ de placa y $\mathrm{ml}$ de pilote (Tabla 7), los costos globales de construcción en pesos colombianos para los sistemas placa-pilote calculados por las diferentes metodologías se muestran en los gráficos de la Figura 31.

De los gráficos de la Figura 31 se observa que para la longitud de $20 \mathrm{~m}$ y diámetros de 0.6 y $0.8 \mathrm{~m}$, el método de elementos de finitos ofrece una solución más económica

d)

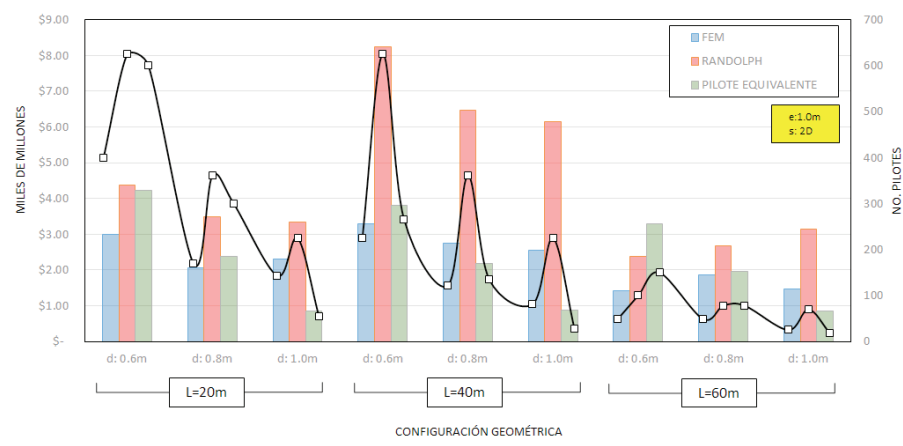

e)

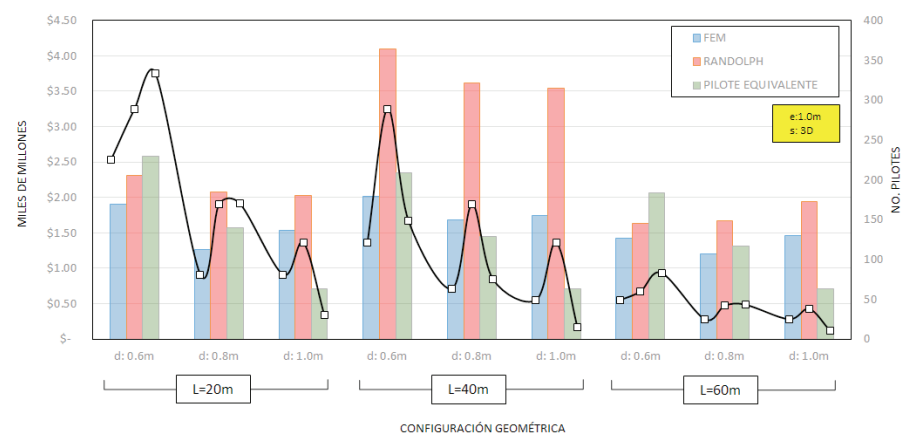

f)

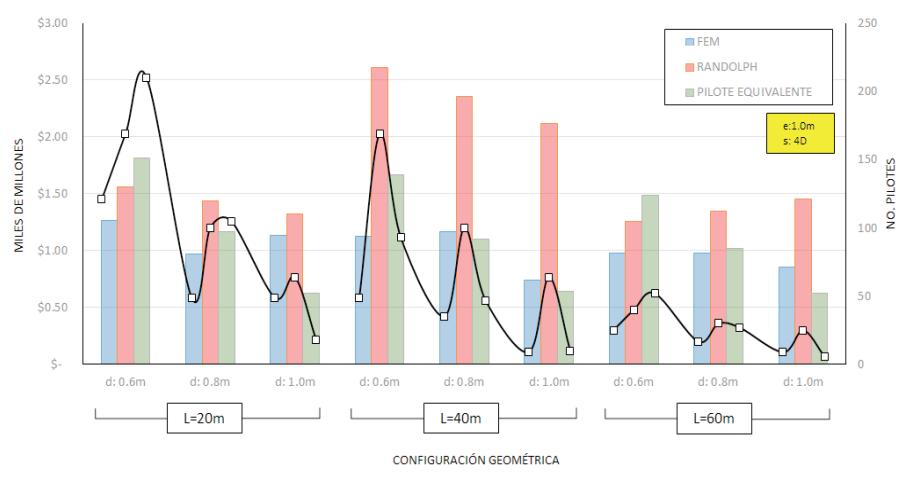

Figura 31: Variación de costos entre metodologías para un espesor de placa de: a) $0.5 \mathrm{~m}$ y $S=2 D$, b) $0.5 \mathrm{~m}$ y $S=3 D$, c) $0.5 \mathrm{~m}$ y $S=$ $4 D$, d) $1 \mathrm{~m}$ y $S=2 D$, e) $1 \mathrm{~m}$ y $S=3 D$ y f) $1 \mathrm{~m}$ y $S=4 D$ 
frente a la del método del pilote equivalente. Cuando el diámetro de los pilotes alcanza su valor máximo de $1 \mathrm{~m}$, la solución más económica resulta ser la del método de pilote equivalente. Este resultado se repite para las longitudes de 40 y $60 \mathrm{~m}$. En general el método de Randolph arroja la solución más costosa en comparación con las otras metodologías de diseño.

\section{Conclusiones}

Se calcularon asentamientos totales para sistemas combinados de cimentación placa + pilotes, utilizando el modelo constitutivo elasto-plástico Mohr-Coulomb, permitiendo la disipación de la presión de poros. Para las condiciones geotécnicas de los suelos presentes en la Sabana de Bogotá (suelos blandos), el asentamiento obtenido para pilotes de $20 \mathrm{~m}$ usando el método de placa equivalente es un $78 \%$ mayor respecto al método de pilote equivalente, $82 \%$ mayor si se compara con los métodos de FEM y Poulos y Davis y 55\% mayor respecto al método de Poulos-Davis Randolph PDR. En el caso de los pilotes de $40 \mathrm{~m}$, la diferencia fue del $66 \%$ respecto al resto de metodologías; y del $45 \%$ para el caso en donde los pilotes tienen $60 \mathrm{~m}$ de longitud. El método de Poulos y Davis bajo la consideración de una distribución de carga $30 \%$ placa y $70 \%$ pilotes, no es aplicable en la búsqueda de un asentamiento de $30 \mathrm{~cm}$ para las condiciones de la Sabana de Bogotá. Hay que suponer que los pilotes deben tomar más del $100 \%$ de la carga (puede llegar hasta el $260 \%$ ). El considerar el avance constructivo de los pisos de una estructura afecta el valor de asentamientos finales esperados para la edificación en un 10\% según lo obtenido en elementos finitos y un $9 \%$ para la metodología de placa equivalente frente al calculado considerando la aplicación de la carga de forma instantánea. Las metodologías tradicionales usadas para el cálculo de los sistemas placapilote, estarían sobreestimando los asentamientos según los casos modelados.

Para las metodologías de Randolph, pilote equivalente y FEM se encontró que la variable geométrica que más incidencia tiene en la reducción de los asentamientos es la longitud de los pilotes, en donde la mayor reducción se presentó cuando se aumentó la longitud de los pilotes de 20 a $40 \mathrm{~m}$. Para lograr maximizar la reducción de los asentamientos diferenciales en los sistemas placa-pilote, el grupo de pilotes debe ocupar el 37 al 59\% del área de la placa, medidos desde el centro. Para las condiciones geotécnicas identificadas en esta investigación y de acuerdo a los resultados de las modelaciones FEM, el considerar una placa rígida, de espesor $1 \mathrm{~m}$, no ofrece ningún tipo de ventaja respecto a la repartición de cargas que existe entre la placa y los pilotes; en donde la diferencia entre ambas es del $4 \%$. Sin embargo, en términos de costos, la placa de mayor espesor ofrece en la mayoría de los casos (74 \%) una solución más económica debido a la reducción del número de pilotes. De acuerdo con los modelos de FEM, el mayor valor de excesos de presiones de poros se presenta en la punta de los pilotes. Con la excepción de grupos de pilotes que ocupen menos del 30\% del área de la placa en cuyo caso los mayores excesos de presión de poros se generan en la parte inferior de la placa. El sistema placa pilote obtenido por el método de Randolph es el más costoso comparado con las otras metodologías. Sin embargo, en términos de asentamientos ofrece una aproximación bastante cercana a los valores reportados en las modelaciones de elementos finitos cuando la longitud de los pilotes es mayor o igual a $40 \mathrm{~m}$.

\section{Referencias}

Bajad, S.P. and Sahu, R.B. (2009). Optimun design of piled raft in soft clay - a model study. Indian Geotechnical Society Conference, Guntuy, 131-134

Bhowmik, R. and Samanta, M. (2013). Numerical analysis of piled-raft foundation under vertical load in stone column improved soil. Proceedings of Indian Geotechnical Conference, Roorkee, 1-10

Cho, J., Lee, J.H., Jeong, S. and Lee, J. (2012). The settlement behavior of piled raft in clay soils. Ocean Engineering 53, 153-163

Cooke, R.W. (1986). Piled raft foundations on stiff clays - a contribution to design philosophy. Géotechnique 36(2), 169-203

Cooke, R.W., Bryden Smith, D.W., Gooch, M.N. and Sillett, D.F. (1981). Some observations of the foundation loading and settlement of a multi-storey building on a piled raft foundation in London clay. Proceedings of the Institution of Civil Engineers 70(3), 433-460

Davids, A., Wongso, J., Popovic, D. and McFarlane, A. (2008). A Postcard from Dubai design and construction of some of the tallest buildings in the world. CTUBH 8th World Congress, Dubai, 3-5 
Desai, C.S., Zaman, M.M., Lightner, J.G. and Siriwardane, H.J. (1984). Thin-layer element for interfaces and joints. International Journal for Numerical and Analytical Methods in Geomechanics 8(1), 19-43

Elwakil, A.Z. and Azzam, W.R. (2016). Experimental and numerical study of piled raft system. Alexandria Engineering Journal 55(1), 547-560

Fattah, M.Y., Al-Mosawi, M.J. and Al-Zayadi, A. A. (2013). Time dependent behavior of piled raft foundation in clayey soil. Geomechanics and Engineering 5(1):17-36

GGS (2014). Empfehlungen des Arbeitskreises Numerik in der Geotechnik - EANG. German Geotechnical Society, WileyVCH Verlag GmbH, Germany

Hansbo, S. and Jendeby, L. (1983). A case study of two alternative foundation principles: conventional friction piling and creep piling. Väg-och Vattenbyggaren 7(8), 29-31

Ibáñez, L.O. (2017). Análisis de la influencia de la profundidad de cimentación en la disminución de asentamientos en losas de fundación combinadas con pilotes. Obras y Proyectos 22, 42-49

Katzenbach, R., Arslan, U., Moorman, C. and Reul, O. (1998). Pile raft foundation: interaction between piles and raft. Darmstadt Geotechnics 4(2), 279-296

Leung, Y.F., Soga, K., Lehane, B.M., and Klar, A. (2010). Role of linear elasticity in pile group analysis and load test interpretation. Journal of Geotechnical and Geoenvironmental Engineering 136(12), 1686-1694

Montaña, D. (2013). Características de compresibilidad y resistencia de arcillas típicas del depósito lacustre de Bogotá. Tesis de maestría, Escuela Colombiana de Ingeniería Julio Garavito, Bogotá, Colombia

Moya, J. y Rodríguez, J. (1987). El subsuelo de Bogotá y los problemas de cimentaciones. $8 v a$ Conferencia Panamericana de Mecánica de Suelos e Ingeniería de Fundaciones, Cartagena de Indias, Colombia, 197-264
NSR-10 (2010). Reglamento Colombiano de construcción sismo resistente. Asociación Colombiana de Ingeniería Sísmica, Colombia

Orozco, L.F. (2006). Asentamientos de fundaciones en la arcilla de Bogotá. IV Encuentro Nacional de Ingenieros de Suelos y Estructuras. Colombia

Palacio, J. (2015). Análisis comparativo de metodologías de diseño de sistemas de cimentación placa-pilote. Tesis de maestría, Escuela Colombiana de Ingeniería Julio Garavito, Bogotá, D.C.

Patil, J.D., Vasanvala, S.A. and Solanki, C.H. (2013). A study on piled raft foundation: state of art. International Journal of Engineering Research and Technology 2(8):1464-1470

Poulos, H.G. (2001). Piled raft foundations: design and applications. Géotechnique 51(2): 95-113

Poulos, H.G. and Davis, E.H. (1980). Pile foundation analysis and design. John Wiley \& Sons

Randolph, M.F. (1994). Design methods for pile groups and piled rafts. $13^{\text {th }}$ International Conference on Soil Mechanics and Geotechnical Engineering, New Delhi, vol. 5, 62-82

Reul, O. and Randolph, M.F. (2003). Piled rafts in overconsolidated clay: comparison of in situ measurements and numerical analyses. Géotechnique 53(3), 301-315

Small, J.C. and Liu, H.L.S. (2008). Time-settlement behaviour of piled raft foundations using infinite elements. Computers and Geotechnics 35(2): 187-195

Tan, Y.C., Chow, C.M. and Gue, S.S. (2005). Piled raft with different pile length for medium-rise buildings on very soft clay. XVI International Conference on Soil Mechanics and Geotechnical Engineering, Osaka, vol. 4, 2045-2048

Tomlinson, M. and Woodward, J. (2014). Pile design and construction practice. 6th edition, CRC Press 